\title{
Neutrino masses from universal fermion mixing
}

\author{
R. Gastmans \\ Institute for Theoretical Physics, Katholieke Universiteit Leuven, B-3001 Leuven, Belgium \\ Per Osland \\ Department of Physics, University of Bergen, Allégaten 55, N-5007 Bergen, Norway
}

Tai Tsun $\mathrm{Wu}$

Gordon McKay Laboratory, Harvard University, Cambridge, Massachusetts 02138 and Theoretical Physics Division, CERN, CH-1211 Geneva 23, Switzerland

(Received 23 October 2002; published 28 March 2003)

\begin{abstract}
If three right-handed neutrinos are added to the standard model, then, for the three known generations, there are six quarks and six leptons. It is then natural to assume that the symmetry considerations that have been applied to the quark matrices are also valid for the lepton mass matrices. Under this assumption, the solar and atmospheric neutrino data can be used to determine the individual neutrino masses. Using the $\chi^{2}$ fit, it is found that the mass of the lightest neutrino is $(2-5) \times 10^{-3} \mathrm{eV}$, that of the next heavier neutrino is $(10-13) \times 10^{-3} \mathrm{eV}$, while the mass of the heaviest neutrino is $(52-54) \times 10^{-3} \mathrm{eV}$.

DOI: 10.1103/PhysRevD.67.053005

PACS number(s): 14.60.Pq, 12.15.Ff, 13.15. $+\mathrm{g}$
\end{abstract}

\section{INTRODUCTION}

In the work of Lehmann, Newton and $\mathrm{Wu}$ [1], the Cabibbo-Kobayashi-Maskawa (CKM) [2] matrix is expressed in terms of the masses of the three generations of quarks:

$$
\left(\begin{array}{l}
u \\
d
\end{array}\right)\left(\begin{array}{l}
c \\
s
\end{array}\right)\left(\begin{array}{l}
t \\
b
\end{array}\right) .
$$

This is accomplished by introducing a new horizontal symmetry. Some of the earlier attempts in this direction are given in $[3,4]$, while some of the more recent work on this topic is to be found in $[5,6]$, for example.

Recent experiments at Super-Kamiokande [7,8] indicate the presence of neutrino oscillations, which would imply that the neutrinos are not all massless. If it is accepted that the neutrinos are not massless, then it is most natural in the standard model [9] to introduce three right-handed neutrinos in addition to the three known left-handed ones. In this way, there are six quarks and six leptons. For recent reviews of neutrino physics, see [10]. Most of the recent work on neutrino masses is focused on the ideas of grand unification and the seesaw mechanism (see, e.g., [11]).

In this paper, the consequences of universal quark-lepton mixing are studied. While many authors favor Majorana masses for the neutrinos, this universality is most natural if they instead have Dirac masses. ${ }^{1}$ In other words, the method of Ref. [1] is used to express the lepton CKM matrix and the neutrino mixing matrix in terms of the masses of the three generations of leptons:

\footnotetext{
${ }^{1}$ As shown in [12], neutrino-oscillation experiments cannot distinguish between massive Majorana and Dirac neutrinos.
}

$$
\left(\begin{array}{c}
\nu_{e} \\
e
\end{array}\right)\left(\begin{array}{c}
\nu_{\mu} \\
\mu
\end{array}\right)\left(\begin{array}{c}
\nu_{\tau} \\
\tau
\end{array}\right) .
$$

Of course the masses of the three charged leptons are accurately known, leaving as unknown parameters the masses of the three neutrinos. Thus there are three parameters to be determined instead of seven, the three masses plus the four in the lepton CKM matrix.

It is the purpose of this paper to use the data from solar neutrinos [8,13-17] and atmospheric neutrinos [7] to determine the three neutrino masses separately, not only the differences of their squares. As compared with earlier work on this model [18], mixing in the charged lepton sector is also taken into account.

In Secs. II and III, we review the case of quarks and apply the model to the case of leptons. In Sec. IV, the rotation matrix is discussed, and in Sec. V we construct the resulting mixing matrix for charged-current interactions. In Sec. VI we review the relevant formulas for atmospheric neutrino propagation, whereas Secs. VII and VIII are devoted to the threeflavor Mikheyev-Smirnov-Wolfenstein (MSW) problem and solar neutrinos. In Sec. IX we collect the results of the fits to the data, and Sec. X contains a discussion. Some properties of the mass matrix are discussed in Appendix A, whereas Appendixes B and C contain technical details of the analytical solution of the MSW equations.

\section{REVIEW FOR THE CASE OF QUARKS}

For the quark mass matrices, the result of Ref. [1] is

$$
M(d)=\left(\begin{array}{ccc}
0 & d(d) & 0 \\
d(d) & c(d) & b(d) \\
0 & b(d) & a(d)
\end{array}\right)
$$




$$
M(u)=\left(\begin{array}{ccc}
0 & i d(u) & 0 \\
-i d(u) & c(u) & b(u) \\
0 & b(u) & a(u)
\end{array}\right),
$$

with

$$
b^{2}(d)=8 c^{2}(d), \quad b^{2}(u)=8 c^{2}(u) .
$$

The diagonalization of these mass matrices is achieved by the orthogonal matrices $R(d)$ and $R(u)$; explicitly, ${ }^{2}$

$$
\begin{aligned}
M(d)= & R(d) \operatorname{diag}\left(m_{d},-m_{s}, m_{b}\right) R^{\mathrm{T}}(d), \\
M(u)= & \operatorname{diag}(i, 1,1) R(u) \operatorname{diag}\left(m_{u},-m_{c}, m_{t}\right) \\
& \times R^{\mathrm{T}}(u) \operatorname{diag}(-i, 1,1) .
\end{aligned}
$$

In terms of $R(d)$ and $R(u)$, the CKM mixing matrix [2] is written as

$$
V=R^{\mathrm{T}}(u) \operatorname{diag}(-i, 1,1) R(d) .
$$

For both the $u$ quarks and the $d$ quarks, the number of independent parameters in the mass matrix is three. Hence they can be expressed in terms of the three quark masses. The relations are

$$
\begin{gathered}
a+c=S_{1}=m_{3}-m_{2}+m_{1}, \\
8 c^{2}+d^{2}-a c=-S_{2}=m_{3} m_{2}-m_{3} m_{1}+m_{2} m_{1}, \\
a d^{2}=-S_{3}=m_{1} m_{2} m_{3},
\end{gathered}
$$

where $m_{1}, m_{2}, m_{3}$ mean respectively $m_{u}, m_{c}, m_{t}$ and $m_{d}, m_{s}, m_{b}$ for the $u$ and $d$ quarks. Because of the known masses, the inequalities

$$
m_{1} \leqslant m_{2} \leqslant m_{3}
$$

are used throughout the analysis of Ref. [1].

In Ref. [1], it is found that [19]

$$
\left|J_{C P}\right| \simeq 2.6 \times 10^{-5} \text {. }
$$

This is consistent with the experimental value of $(3.0 \pm 1.3) \times 10^{-5}[20]$. This experimental value is expected to improve significantly in the near future.

\section{APPLICATION TO THE CASE OF LEPTONS}

Since the neutrinos are now known to have masses, the symmetry considerations for the quark mass matrices are equally applicable to the leptons. Furthermore, the leptons, not being confined, have masses that are better defined than those of the quarks. Replacing $d$ and $u$ by the charged leptons $\ell$ and the neutrinos $\nu$, Eqs. (2.1), (2.2) and (2.3) take the form

\footnotetext{
${ }^{2}$ Note misprints in Eqs. (27) and (35) of Ref. [1], $i \rightarrow-i$.
}

$$
M(\ell)=\left(\begin{array}{ccc}
0 & d(\ell) & 0 \\
d(\ell) & c(\ell) & b(\ell) \\
0 & b(\ell) & a(\ell)
\end{array}\right),
$$

$$
M(\nu)=\left(\begin{array}{ccc}
0 & i d(\nu) & 0 \\
-i d(\nu) & c(\nu) & b(\nu) \\
0 & b(\nu) & a(\nu)
\end{array}\right),
$$

with

$$
b^{2}(\ell)=8 c^{2}(\ell), \quad b^{2}(\nu)=8 c^{2}(\nu) .
$$

The rotation matrices $R(\ell)$ and $R(\nu)$ are defined in exactly the same manner:

$$
\begin{aligned}
M(\ell)= & R(\ell) \operatorname{diag}\left(m_{e},-m_{\mu}, m_{\tau}\right) R^{\mathrm{T}}(\ell), \\
M(\nu)= & \operatorname{diag}(i, 1,1) R(\nu) \operatorname{diag}\left(m_{1},-m_{2}, m_{3}\right) \\
& \times R^{\mathrm{T}}(\nu) \operatorname{diag}(-i, 1,1),
\end{aligned}
$$

where in Eq. (3.5) the masses of the three neutrinos are designated as $m_{1}, m_{2}$, and $m_{3}$. The lepton CKM mixing matrix is

$$
V^{\ell}=R^{\mathrm{T}}(\nu) \operatorname{diag}(-i, 1,1) R(\ell) .
$$

There are actually significant differences between the quark case and the lepton case.

(A) In the quark case, that there is $C P$ violation has been known for many years [21,22]. In the lepton case, it is not known whether $C P$ is conserved or not. While it is tempting, on the basis of quark-lepton universality, to believe that $C P$ nonconservation also holds for leptons, the possibility of lepton $C P$ conservation cannot be excluded. In the former case, Eq. (3.2) holds; in the latter case, both $i$ and $-i$ on the right-hand side of Eq. (3.2) are replaced by 1. To take both possibilities into account, Eq. (3.2) needs to be generalized to

$$
M(\nu)=\left(\begin{array}{ccc}
0 & \epsilon d(\nu) & 0 \\
\epsilon^{*} d(\nu) & c(\nu) & b(\nu) \\
0 & b(\nu) & a(\nu)
\end{array}\right),
$$

where the two cases above correspond to $\epsilon=i, 1$, respectively. Similarly, Eqs. (3.5) and (3.6) take the forms

$$
\begin{aligned}
M(\nu)= & \operatorname{diag}(\epsilon, 1,1) R(\nu) \operatorname{diag}\left(m_{1},-m_{2}, m_{3}\right) \\
& \times R^{\mathrm{T}}(\nu) \operatorname{diag}\left(\epsilon^{*}, 1,1\right),
\end{aligned}
$$

and

$$
V^{\ell}=R^{\mathrm{T}}(\nu) \operatorname{diag}\left(\epsilon^{*}, 1,1\right) R(\ell) .
$$

(B) In the case of quarks, both the masses and the absolute values of the elements of the CKM mixing matrix are known experimentally. In the case of the leptons, there is much less experimental information. While the masses of the 
three charged leptons are accurately known, the corresponding knowledge about the neutrinos is limited to differences between the masses squared, $m_{1}^{2}, m_{2}^{2}, m_{3}^{2}$. It is for this reason that the lepton case may be considered to be more challenging, and one of the first tasks is to determine the individual masses from the existing experimental data. This is to be carried out in Sec. IX.

(C) The lack of knowledge about the neutrino masses has another profound consequence. For quarks, the inequality (2.8) holds for both $u$ and $d$. In contrast, because of the presence of the minus sign with $m_{2}$ in Eq. (3.5), all that is known about neutrino masses is

$$
m_{1} \leqslant m_{3}
$$

In other words, for neutrinos, Eq. (3.10) can be used, but not Eq. (2.8). The first task is therefore to determine the allowed region in the space $\left(m_{1}, m_{2}, m_{3}\right)$ of neutrinos, which must be between those permitted by Eqs. (2.8) and (3.10).

It follows from Eq. (2.7) that the parameter $a$, which must be positive, satisfies the cubic equation

$$
9 a^{3}-17 S_{1} a^{2}+\left(8 S_{1}^{2}+S_{2}\right) a-S_{3}=0 .
$$

Any real cubic equation can have either one or three real solutions. Where there is one real solution, that one is negative, and thus unphysical, as is seen from Eq. (2.7). Where there are three real solutions, one of them is negative, while two are positive. We shall refer to these two positive solutions as solution 1 (larger $a$ ) and solution 2 (smaller $a$ ).

It is instructive to consider briefly the simple case $m_{1}$ $=m_{2}=0$. In this case, it follows from Eq. (2.7) that $S_{1}$ $=m_{3}$ and $S_{2}=S_{3}=0$, and Eq. (3.11) reduces to

$$
9 a^{3}-17 m_{3} a^{2}+8 m_{3}^{2} a=0
$$

with the solutions

$$
a=m_{3}, \quad \frac{8}{9} m_{3}, \quad 0 .
$$

Here 0 is the limiting value of the negative solution and hence is of no interest. From the above definitions, in this case $a=m_{3}$ is solution 1 while $a=\frac{8}{9} m_{3}$ is solution 2. From Eq. (3.7), the mass matrices are

$$
M(\nu)=\left(\begin{array}{lll}
0 & 0 & 0 \\
0 & 0 & 0 \\
0 & 0 & 1
\end{array}\right) m_{3},
$$

for solution 1 , and

$$
M(\nu)=\left(\begin{array}{ccc}
0 & 0 & 0 \\
0 & \frac{1}{9} & \frac{2 \sqrt{2}}{9} \\
0 & \frac{2 \sqrt{2}}{9} & \frac{8}{9}
\end{array}\right) m_{3},
$$

for solution 2 .

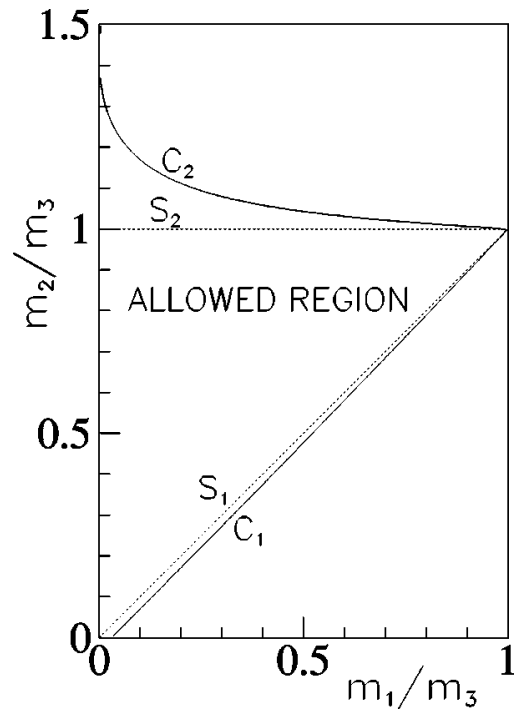

FIG. 1. The allowed region for the three neutrino masses $m_{1}$, $m_{2}$, and $m_{3}$ is bounded by parts of the axes and the solid curves $\mathcal{C}_{1}$ and $\mathcal{C}_{2}$. The lines $\mathcal{S}_{1}$ and $\mathcal{S}_{2}$ correspond to $m_{1}=m_{2}$ and $m_{2}=m_{3}$, respectively.

In Ref. [1] for the quark mass matrices, only solution 1 was considered.

The above considerations on the cubic equation (3.11) can be used to determine the allowed physical region in the $\left(m_{1} / m_{3}, m_{2} / m_{3}\right)$ plane, as shown in Fig. 1. This region is only slightly larger than the triangle given by the inequality (2.8), with two additional regions, one where $m_{2}>m_{3}$ and the other a very small one with $m_{1}>m_{2}$

The following notation is convenient.

(1) $\mathcal{R}$ denotes the entire allowed region, not including the boundary, for the three neutrino masses $m_{1}, m_{2}$, and $m_{3}$, as shown in Fig. 1.

(2) $\mathcal{R}_{0}$ denotes the part of $\mathcal{R}$ where $m_{1}<m_{2}<m_{3}$.

(3) $\mathcal{R}_{1}$ denotes the part of $\mathcal{R}$ where $m_{1}>m_{2}$.

(4) $\mathcal{R}_{2}$ denotes the part of $\mathcal{R}$ where $m_{2}>m_{3}$.

(5) $\mathcal{S}_{1}$ denotes the part of $\mathcal{R}$ where $m_{1}=m_{2}$; similarly $\mathcal{S}_{2}$ the part where $m_{2}=m_{3}$.

Thus

$$
\mathcal{R}=\mathcal{R}_{0}+\mathcal{R}_{1}+\mathcal{R}_{2}+\mathcal{S}_{1}+\mathcal{S}_{2}
$$

(6) $\mathcal{C}_{1}$ is the curved part of the boundary of $\mathcal{R}_{1}$.

(7) $\mathcal{C}_{2}$ is the curved part of the boundary of $\mathcal{R}_{2}$.

(8) $\mathcal{C}_{3}$ is the plane

$$
0<m_{2} / m_{3}<(17+\sqrt{33}) / 16 \simeq 1.42, \quad m_{1}=0 .
$$

(9) $\mathcal{C}_{4}$ is the plane

$$
0<m_{1} / m_{3}<17-12 \sqrt{2} \simeq 0.029, \quad m_{2}=0 .
$$

\section{THE ROTATION MATRIX}

There is an elementary but somewhat complicated issue of the sign ambiguities in the definition of the rotation matrices $R(\nu)$ and $R(\ell)$ as given by Eqs. (3.4) and (3.5). 
It is seen from Eq. (2.7) that, for either solution 1 or solution 2 as defined in Sec. III, the values of $m_{1}, m_{2}$, and $m_{3}$ in $\mathcal{R}$ determine those of $a, b^{2}$, and $d^{2}$, but not $b$ and $d$. Therefore, for either $R(\nu)$ or $R(\ell)$, there are actually eight distinct $R$ 's in $\mathcal{R}: R^{k \pm \pm}$, where $k=1$ for solution 1 and $k$ $=2$ for solution 2 , and where the first \pm and the second \pm designate the "parities" (or sign factors) of $b$ and $d$ respectively (see below).

Since these $R$ 's are determined by

$$
\left(\begin{array}{lll}
0 & d & 0 \\
d & c & b \\
0 & b & a
\end{array}\right) R=R\left(\begin{array}{ccc}
m_{1} & 0 & 0 \\
0 & -m_{2} & 0 \\
0 & 0 & m_{3}
\end{array}\right),
$$

the elements of $R$ are given explicitly by

$$
\begin{aligned}
& R_{11}=d\left(m_{1}-a\right) y_{1}, \quad R_{12}=-d\left(m_{2}+a\right) y_{2}, \\
& R_{13}=d\left(m_{3}-a\right) y_{3}, \quad R_{21}=m_{1}\left(m_{1}-a\right) y_{1}, \\
& R_{22}=m_{2}\left(m_{2}+a\right) y_{2}, \quad R_{23}=m_{3}\left(m_{3}-a\right) y_{3}, \\
& R_{31}=b m_{1} y_{1}, \quad R_{32}=-b m_{2} y_{2}, \quad R_{33}=b m_{3} y_{3},
\end{aligned}
$$

where the values of $y_{1}, y_{2}$, and $y_{3}$ are such that $R$ is orthogonal.

This condition of orthogonality does not determine the signs of these $y$ 's:

$$
y_{j}=\frac{ \pm 1}{\sqrt{d^{2}\left(\lambda_{j}-a\right)^{2}+\lambda_{j}^{2}\left(\lambda_{j}-a\right)^{2}+b^{2} \lambda_{j}^{2}}},
$$

where

$$
\lambda_{1}=m_{1}, \quad \lambda_{2}=-m_{2}, \quad \lambda_{3}=m_{3} .
$$

The entire problem is to choose the three \pm signs in Eq. (4.3).

Strictly speaking, any choice of sign will do. Since such choices lead to a large number of possible CKM mixing matrices for leptons (for each $R$, there are $2^{3}=8$ possible choices of signs), it is useful to make an intelligent choice of these signs.

The basic principle to be used to choose the signs is that of continuity, i.e., the continuity of the nine $R_{i j}$ for each $R$. For example, each of these $R_{i j}$ must be continuous in $\mathcal{R}$. Note that the continuity of an $R$ implies the continuity of its derivatives with respect to the masses $m_{1}, m_{2}$, and $m_{3}$.

The problem to be solved is to find a set of eight $3 \times 3$ matrices $R^{k \pm \pm}$ with the following conditions: (1) $R^{k \pm \pm}$ are continuous in $\mathcal{R}$; (2) $R_{j j}^{k \pm \pm}>0$ in $\mathcal{R}_{0}$; and (3) $R^{1 \pm \pm}$ $=R^{2 \pm \pm}$ on $\mathcal{C}_{1}$.

Condition (3) requires the following comments. First, this condition can be imposed on $\mathcal{C}_{1}$ or $\mathcal{C}_{2}$, but not on $\mathcal{C}_{3}$ or $\mathcal{C}_{4}$. The reason is that, on $\mathcal{C}_{1}$ and $\mathcal{C}_{2}$, the values of $a$ for the first and second solutions are the same. Secondly, what this equation means is that, given an $R^{1}$, for example $R^{1++}$, there is a choice for the two \pm signs for $R^{2 \pm \pm}$ so that the $R$ 's are equal; this can only be achieved on either $\mathcal{C}_{1}$ or $\mathcal{C}_{2}$, not on
TABLE I. Signs of the coefficients of the cubic equation (4.5).

\begin{tabular}{ccccc}
\hline \hline & 9 & $-10 S_{1}$ & $S_{1}^{2}+S_{2}$ & $-S_{1} S_{2}+S_{3}$ \\
\hline $\mathcal{R}_{2}$ & + & \pm & - & - \\
$\mathcal{R}_{0}$ & + & - & \pm & + \\
$\mathcal{R}_{1}$ & + & - & + & - \\
\hline \hline
\end{tabular}

both. Actually, there is no such choice: If $\mathcal{C}_{2}$ is chosen, no solution exists because of the condition (2). It is therefore necessary to impose this condition on $\mathcal{C}_{1}$, for any " $b$ parity" and " $d$ parity." This is already indicated in condition (3) above.

The "parities" or sign factors are defined as follows: For positive " $b$ parity," $b$ has the opposite sign of $c$, which is the convention of [1]. In particular, for solution $1, b$ is then positive in $\mathcal{R}_{0}$. When the " $b$ parity" is flipped, $R_{13}^{k}, R_{23}^{k}, R_{31}^{k}$, and $R_{32}^{k}$ change sign. The " $d$ parity" is the sign of $d$ in all of $\mathcal{R}$. When this sign is flipped, $R_{12}^{k}, R_{13}^{k}, R_{21}^{k}$, and $R_{31}^{k}$ change sign.

Attention is next turned to the cubic equation (3.12) for $a$. Since $c=S_{1}-a$ from Eq. (2.7), the corresponding cubic equation for $c$ is

$$
9 c^{3}-10 S_{1} c^{2}+\left(S_{1}^{2}+S_{2}\right) c-\left(S_{1} S_{2}-S_{3}\right)=0 .
$$

The nice formula

$$
S_{3}-S_{1} S_{2}=\left(m_{2}-m_{1}\right)\left(m_{3}-m_{2}\right)\left(m_{3}+m_{1}\right)
$$

shows that $S_{3}-S_{1} S_{2}$ is positive in $\mathcal{R}_{0}$, and in fact implies that, in $\mathcal{R}_{0}$, two of the solutions $c$ from Eq. (4.5) are positive, while one is negative. In a similar way, the signs of the coefficients of the cubic equation (4.5) are listed in Table I.

Since the three solutions of this cubic equation are known to be all real in $\mathcal{R}$, Table II follows immediately from Table I. In particular

$$
\begin{aligned}
& c=0 \text { on } \mathcal{S}_{1} \text { for solution } 1, \\
& c=0 \text { on } \mathcal{S}_{2} \text { for solution } 2 .
\end{aligned}
$$

The next task is to show that

$$
a-m_{1} \geqslant 0
$$

in $\mathcal{R}$, where the equality sign holds only on $\mathcal{S}_{2}$, and then only for solution 2 . Similarly,

$$
m_{3}-a \geqslant 0
$$

TABLE II. Signs of $c$ for solution 1 and solution 2 .

\begin{tabular}{ccc}
\hline \hline & Solution 1 & Solution 2 \\
\hline $\mathcal{R}_{2}$ & - & - \\
$\mathcal{R}_{0}$ & - & + \\
$\mathcal{R}_{1}$ & + & + \\
\hline \hline
\end{tabular}


Solution $1++$
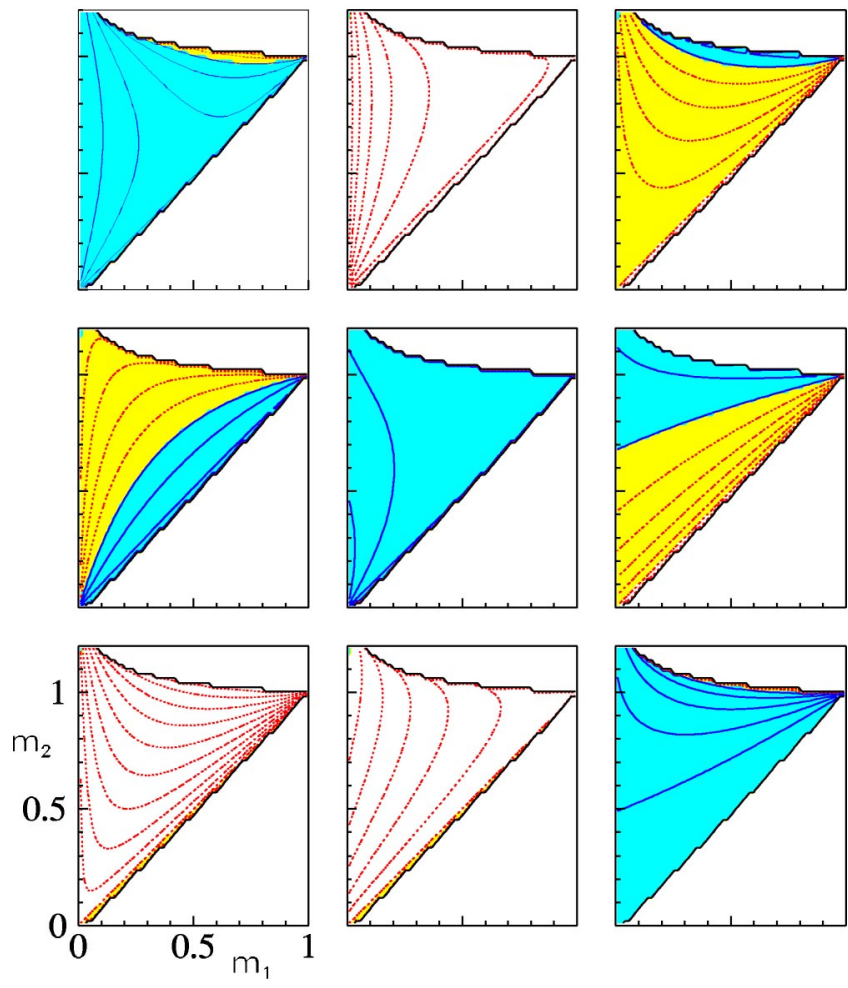

FIG. 2. Solution 1: $R_{i j}^{1++}(\nu)$ matrix elements vs $m_{1}$ and $m_{2}\left(m_{3}=1\right)$, for $i=1,2,3$ and $j=1,2,3$. The dark regions indicate where the values exceed 0.5 , and the lightly shaded regions indicate where the values are between 0 and 0.5 . In the unshaded (white) regions, the values are negative.

in $\mathcal{R}$, where the equality sign holds only on $\mathcal{S}_{1}$, and then only for solution 1 . These derivations are straightforward and hence omitted. Note that the left-hand sides of these inequalities play an important role in Eq. (4.2). The two relations may conveniently be summarized as

$$
m_{1} \leqslant a \leqslant m_{3}
$$

It is also useful to note that

$$
m_{\mathcal{S}_{1}, \text { sol } 1}=|d|, \quad|d|_{\mathcal{S}_{2}, \text { sol } 2}=m_{3},
$$

but in this latter case, these are not extrema.

With this knowledge, the resulting signs are easily determined as given in Figs. 2 and 3. The signs of $y_{j}$, respecting conditions (1), (2), and (3) above, are as follows:

For solution 1 , some $R_{i j}^{1 \pm \pm}$ vanish on $\mathcal{S}_{1}$. They are $i j$ $=13,23,31$, and 32 , corresponding to $m_{3}-a$ and $b$ becoming zero. (These $R_{i j}^{1 \pm \pm}$ thus have opposite signs in $\mathcal{R}_{0}$ and $\mathcal{R}_{1}$.)

For solution 2 , some $R_{i j}^{2 \pm \pm}$ vanish on $\mathcal{S}_{2}$. They are $i j$ $=11,21,32$, and 33, corresponding to $a-m_{1}$ and $b$ becoming zero. (These $R_{i j}^{2 \pm \pm}$ thus have opposite signs in $\mathcal{R}_{0}$ and $\mathcal{R}_{2}$.)

\section{Solution $2++$}
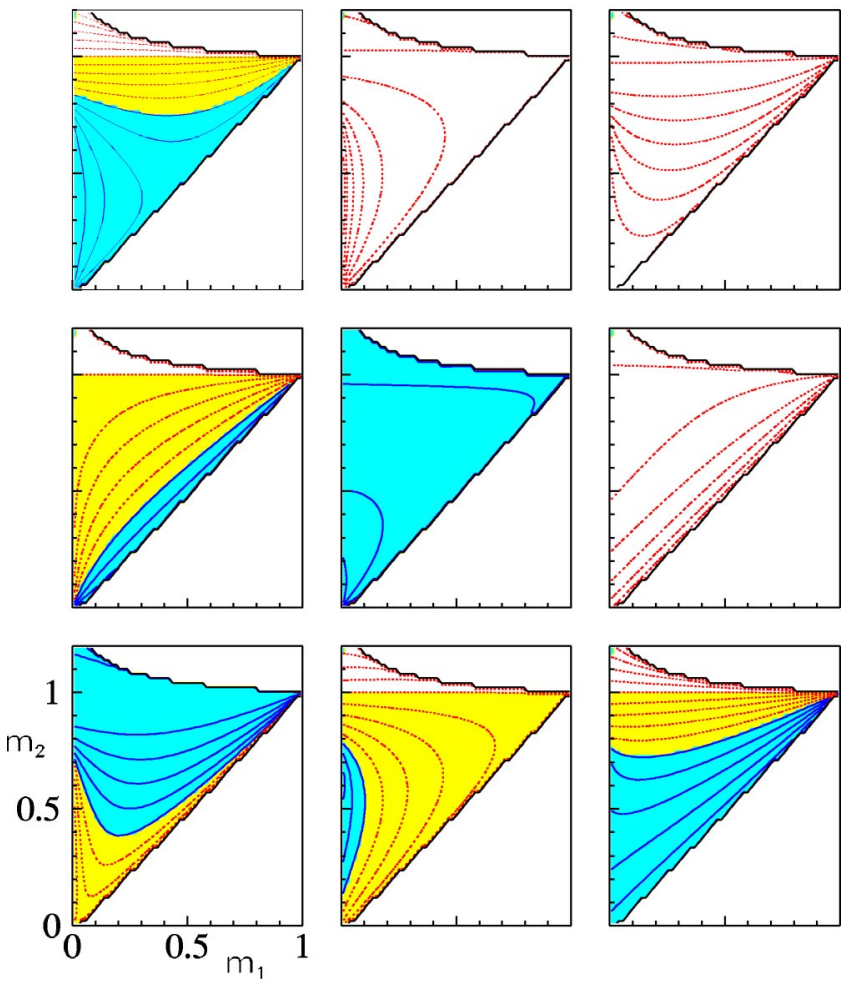

FIG. 3. Solution 2: $R_{i j}^{2++}(\nu)$ matrix elements vs $m_{1}$ and $m_{2}\left(m_{3}=1\right)$. Compare with Fig. 2 .

In order to have $R_{i j}^{k \pm \pm}$ that are continuous, it is required to flip signs of some $y_{j}$ as these boundaries $\mathcal{S}_{1}$ and $\mathcal{S}_{2}$ are crossed.

The mixing matrices display strong variations with $m_{1}$ and $m_{2}$. For solution 1 , the diagonal elements dominate in much of the parameter space, whereas for solution 2, this is not the case.

For the charged leptons, the masses are strongly hierarchical. Thus, the rotation matrices correspond to the lower lefthand corners of those displayed in Figs. 2 and 3. For solution 1 , this is close to the unit matrix, whereas for solution 2 certain nondiagonal elements are also significant.

\section{THE MIXING MATRIX}

We write the unitary (but not necessarily real) mixing matrix as

$$
U=\left[\begin{array}{ccc}
U_{e 1} & U_{e 2} & U_{e 3} \\
U_{\mu 1} & U_{\mu 2} & U_{\mu 3} \\
U_{\tau 1} & U_{\tau 2} & U_{\tau 3}
\end{array}\right]
$$

where [cf. Eq. (3.9)]

$$
U=\left(V^{\ell}\right)^{\dagger}=R^{\mathrm{T}}(\ell) \operatorname{diag}(\epsilon, 1,1) R(\nu)
$$

relates the neutrino mass eigenstates to the flavor states:

$$
\left|\nu_{e}\right\rangle=U_{e 1}\left|\nu_{1}\right\rangle+U_{e 2}\left|\nu_{2}\right\rangle+U_{e 3}\left|\nu_{3}\right\rangle
$$



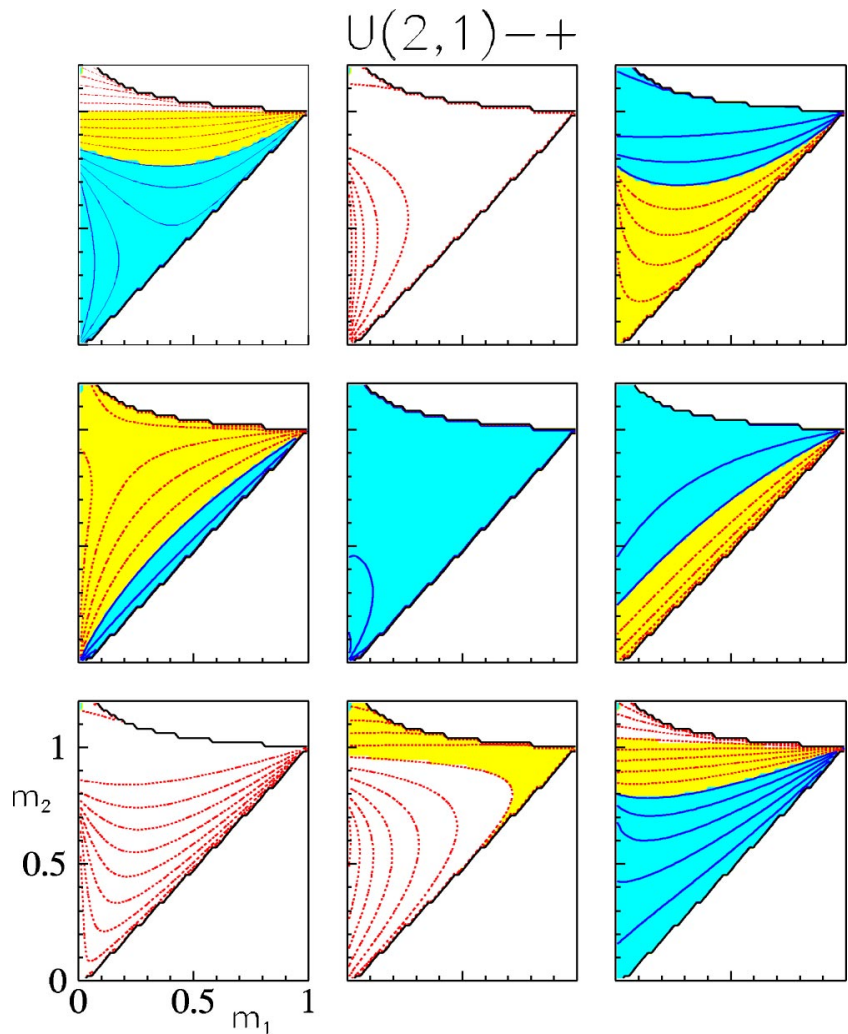

FIG. 4. Mixing matrix $[U(2,1)-+]_{i j}$ vs $m_{1}$ and $m_{2}\left(m_{3}=1\right)$, for $i=1,2,3$ and $j=1,2,3$. The dark regions indicate where the values exceed 0.5 , and the lightly shaded regions indicate where the values are between 0 and 0.5 . In the unshaded (white) regions, the values are negative.

etc. These are the states which enter in charged-current interactions.

We shall label these mixing matrices by the solutions $(1$ or 2) involved in the rotation matrices $R(\nu)$ and $R(\ell)$, and the "parities" associated with the parameters $b$ and $d$, as discussed in Sec. IV. Actually, only the product of the " $b$ parities" and that of the " $d$ parities" matter. Thus it is convenient to define

$$
\begin{aligned}
& b \text { parity }=(b \text { parity })_{\nu} \times(b \text { parity })_{\ell}, \\
& d \text { parity }=(d \text { parity })_{\nu} \times(d \text { parity })_{\ell} .
\end{aligned}
$$

Therefore, there are a total of

$$
2 \times 2 \times 2 \times 2 \times 2
$$

different $U$ matrices, where two factors of 2 arise from the two solutions for the $\nu$ and $\ell$ sectors, two factors of 2 arise from the " $b$ parities" and the " $d$ parities," and the last factor of 2 arises from the choice of $C P$ conservation or $C P$ nonconservation $(\epsilon=1$ or $\epsilon=i)$.

For a representative case, solution 2 for $R(\nu)$ and solution 1 for $R(\ell), b$ parity $=-, d$ parity $=+$, and no $C P$ violation, we show in Fig. 4 the resulting mixing matrix $U(2,1)-+$. Since, for the charged leptons, solution 1 is close to the unit matrix, the resulting $U(2,1)-+$ is rather similar to the corresponding $R(\nu)$ for solution 2 and parities -+ .

\section{ATMOSPHERIC NEUTRINOS}

Let us next review the oscillation formulas that are relevant for atmospheric neutrinos. A neutrino state, which was a pure $\nu_{\alpha}$ state at $t=0$, will at time $t$ have the form

$$
\nu_{\alpha}(t)=\sum_{j=1}^{3} U_{\alpha j} e^{-i E_{j} t} \nu_{j} \quad(\alpha=e, \mu, \tau) .
$$

For relativistic neutrinos, the energy is given as $E_{j}$ $\simeq p+m_{j}^{2} / 2 E$, and the survival probability for the $\nu_{\mu}$ takes the form

$$
\begin{aligned}
P_{\nu_{\mu} \rightarrow \nu_{\mu}}(t)= & 1-4\left[\left|U_{\mu 1}\right|^{2}\left|U_{\mu 2}\right|^{2} \sin ^{2}\left(\frac{\Delta m_{21}^{2} t}{4 E}\right)\right. \\
& +\left|U_{\mu 1}\right|^{2}\left|U_{\mu 3}\right|^{2} \sin ^{2}\left(\frac{\Delta m_{31}^{2} t}{4 E}\right) \\
& \left.+\left|U_{\mu 2}\right|^{2}\left|U_{\mu 3}\right|^{2} \sin ^{2}\left(\frac{\Delta m_{32}^{2} t}{4 E}\right)\right],
\end{aligned}
$$

where $\Delta m_{i j}^{2}=m_{i}^{2}-m_{j}^{2}$.

After propagation over some distance, a neutrino of a different flavor may appear. The probability amplitude for the transition $\alpha \rightarrow \beta$ is given by

$$
\left\langle\nu_{\beta}(t) \mid \nu_{\alpha}(0)\right\rangle=\sum_{k} U_{\beta k}^{*} U_{\alpha k} e^{i m_{k}^{2} t / 2 E} .
$$

In contrast to the survival probability, this expression is not invariant under complex conjugation of $U$.

A case of particular interest is

$$
\begin{aligned}
P_{\nu_{\mu} \rightarrow \nu_{\tau}}(t)= & \left|\sum_{k} U_{\tau k}^{*} U_{\mu k} e^{i m_{k}^{2} t / 2 E}\right|^{2} \\
= & \left\{-4 \operatorname{Re}\left[U_{\tau 1}^{*} U_{\mu 2}^{*} U_{\tau 2} U_{\mu 1}\right] \sin ^{2}\left(\frac{\Delta m_{21}^{2} t}{4 E}\right)\right. \\
& \left.-2 \operatorname{Im}\left[U_{\tau 1}^{*} U_{\mu 2}^{*} U_{\tau 2} U_{\mu 1}\right] \sin \left(\frac{\Delta m_{21}^{2} t}{2 E}\right)\right\} \\
& + \text { cyclic, }
\end{aligned}
$$

where we have used the orthogonality, $\Sigma_{k} U_{\tau k}^{*} U_{\mu k}=0$. For the real case, this simplifies to 


$$
\begin{aligned}
P_{\nu_{\mu} \rightarrow \nu_{\tau}}(t)= & -4 U_{\tau 1} U_{\mu 2} U_{\tau 2} U_{\mu 1} \sin ^{2}\left(\frac{\Delta m_{21}^{2} t}{4 E}\right) \\
& -4 U_{\tau 2} U_{\mu 3} U_{\tau 3} U_{\mu 2} \sin ^{2}\left(\frac{\Delta m_{32}^{2} t}{4 E}\right) \\
& -4 U_{\tau 3} U_{\mu 1} U_{\tau 1} U_{\mu 3} \sin ^{2}\left(\frac{\Delta m_{13}^{2} t}{4 E}\right) .
\end{aligned}
$$

It is instructive to study the simple limit

$$
\left|\Delta m_{21}^{2}\right| \ll\left|\Delta m_{32}^{2}\right|, \quad \Delta m_{21}^{2} t / 4 E \ll 1,
$$

with all $U_{\alpha k}=\mathcal{O}(1)$. Then, by unitarity, Eq. (6.5) simplifies to the familiar expression

$$
P_{\nu_{\mu} \rightarrow \nu_{\tau}}(t) \simeq 4\left|U_{\mu 3}\right|^{2}\left|U_{\tau 3}\right|^{2} \sin ^{2}\left(\frac{\Delta m_{32}^{2} t}{4 E}\right) .
$$

Fitting the data within a two-flavor model, with $\sin ^{2} \theta$ and $\Delta m^{2}$ as independent parameters, one finds [7] large mixing angles. In the limit of Eq. (6.6), this corresponds to large values for $\left|U_{\mu 3} U_{\tau 3}\right|^{2}$; see Eq. (6.7).

The observed suppression of atmospheric $\nu_{\mu}$ [7] suggests masses of the order $m \simeq 0.05 \mathrm{eV}$. In order to determine the neutrino masses, we formulate a $\chi^{2}$ by comparing predicted $\nu_{\mu}$ and $\nu_{e}$ fluxes with data:

$$
\chi_{\mathrm{atm}}^{2}=\sum_{i} \frac{\left(\Phi_{i}-\Phi_{i}^{\exp }\right)^{2}}{\sigma_{i}^{2}} .
$$

The experimental data used are those from SuperKamiokande [7]: the 8 data points (bins in $E / L$, where $L$ $=c t$ ) for $\nu_{\mu}$ and the 8 data points for $\nu_{e}$. These sixteen data points are treated as separate inputs, allowing an overall normalization constant for the two sets of data. Also, since the various survival and transition probabilities are rather sensitive to the precise values of energy and oscillation length, we averaged over these, within each of the 8 bins.

We show in Fig. 5 the contributions to $\chi^{2}$ from the atmospheric-neutrino data, for the mixing matrices $U(2,1)-+$ corresponding to $C P$ conservation (left part) and $C P$ nonconservation (right part). The figure shows $\chi^{2}$ as a function of $m_{1}$ and $m_{2}$, for fixed $m_{3}=0.05 \mathrm{eV}$.

The different solutions and parities that determine the mixing matrices give rather differently shaped $\chi^{2}$ minima when plotted vs $m_{1}$ and $m_{2}$. For most cases, the minima occur inside the region $\mathcal{R}_{0}$. For others, they occur near $\mathcal{S}_{1}$ or near $\mathcal{S}_{2}$.

Comparing with Figs. 2 and 3, we see that solution 1 provides large mixing for $m_{2}$ being a sizable fraction of $m_{3}$, whereas solution 2 favors relatively smaller values of $m_{2}$, or $m_{2}$ close to $m_{1}$.

\section{THE THREE-FAMILY MSW MECHANISM}

The coupled equations satisfied by the three neutrino wave functions are [23]

$$
\begin{aligned}
i \frac{d}{d r}\left(\begin{array}{l}
\phi_{1}(r) \\
\phi_{2}(r) \\
\phi_{3}(r)
\end{array}\right)= & {\left[\left(\begin{array}{ccc}
D(r) & 0 & 0 \\
0 & 0 & 0 \\
0 & 0 & 0
\end{array}\right)\right.} \\
& \left.+\frac{1}{2 p}\left(\begin{array}{lll}
M_{11}^{2} & M_{12}^{2} & M_{13}^{2} \\
M_{21}^{2} & M_{22}^{2} & M_{23}^{2} \\
M_{31}^{2} & M_{32}^{2} & M_{33}^{2}
\end{array}\right)\right]\left(\begin{array}{c}
\phi_{1}(r) \\
\phi_{2}(r) \\
\phi_{3}(r)
\end{array}\right),
\end{aligned}
$$

where $D(r)=\sqrt{2} G_{\mathrm{F}} N_{e}(r)$, with $G_{\mathrm{F}}$ the Fermi weakinteraction constant and $N_{e}(r)$ the solar electron density at a distance $r$ from the center of the Sun. Furthermore, we denote the flavor states $\nu_{e}=\phi_{1}, \nu_{\mu}=\phi_{2}, \nu_{\tau}=\phi_{3}$. These are the states which enter in charged-current interactions.

The evolution of the neutrino wave functions is determined by the squared mass matrix,

$$
\begin{aligned}
{[M(\nu)]^{2} } & =\left(\begin{array}{ccc}
d^{2} & \epsilon c d & \epsilon b d \\
\epsilon^{*} c d & b^{2}+c^{2}+d^{2} & b(a+c) \\
\epsilon^{*} b d & b(a+c) & a^{2}+b^{2}
\end{array}\right) \\
& \equiv\left(\begin{array}{lll}
M_{11}^{2} & M_{12}^{2} & M_{13}^{2} \\
M_{21}^{2} & M_{22}^{2} & M_{23}^{2} \\
M_{31}^{2} & M_{32}^{2} & M_{33}^{2}
\end{array}\right)
\end{aligned}
$$

the neutrino momentum, $p$, and the solar electron density. Here, $M_{i j}^{2} \equiv\left(M^{2}\right)_{i j}$, and $\epsilon=1$ ( $C P$ conservation) or $\epsilon=i$ (CP nonconservation).

It is actually a good approximation to take an exponential electron density, $N_{e}(r)=N_{e}(0) \exp \left(-r / r_{0}\right)$. A fit to the solar density as given by [27] gives $r_{0}=6.983 \times 10^{4} \mathrm{~km}$. For this case of an exponential solar density, the three-component wave equation can be solved in terms of generalized hypergeometric functions, ${ }_{2} F_{2}$ [24].

The case treated in [24] was that of a real mass matrix. In that case, by scaling and shifting the radial variable, $u$ $=r / r_{0}+u_{0}$, with $u_{0}$ determined such that

$$
D(0) r_{0} e^{u_{0}}=1,
$$

Eq. (7.1) could be transformed into the form

$$
i \frac{d}{d u}\left[\begin{array}{l}
\psi_{1}(u) \\
\psi_{2}(u) \\
\psi_{3}(u)
\end{array}\right]=\left[\begin{array}{ccc}
\omega_{1}+e^{-u} & \chi_{2} & \chi_{3} \\
\chi_{2} & \omega_{2} & 0 \\
\chi_{3} & 0 & \omega_{3}
\end{array}\right]\left[\begin{array}{l}
\psi_{1}(u) \\
\psi_{2}(u) \\
\psi_{3}(u)
\end{array}\right],
$$



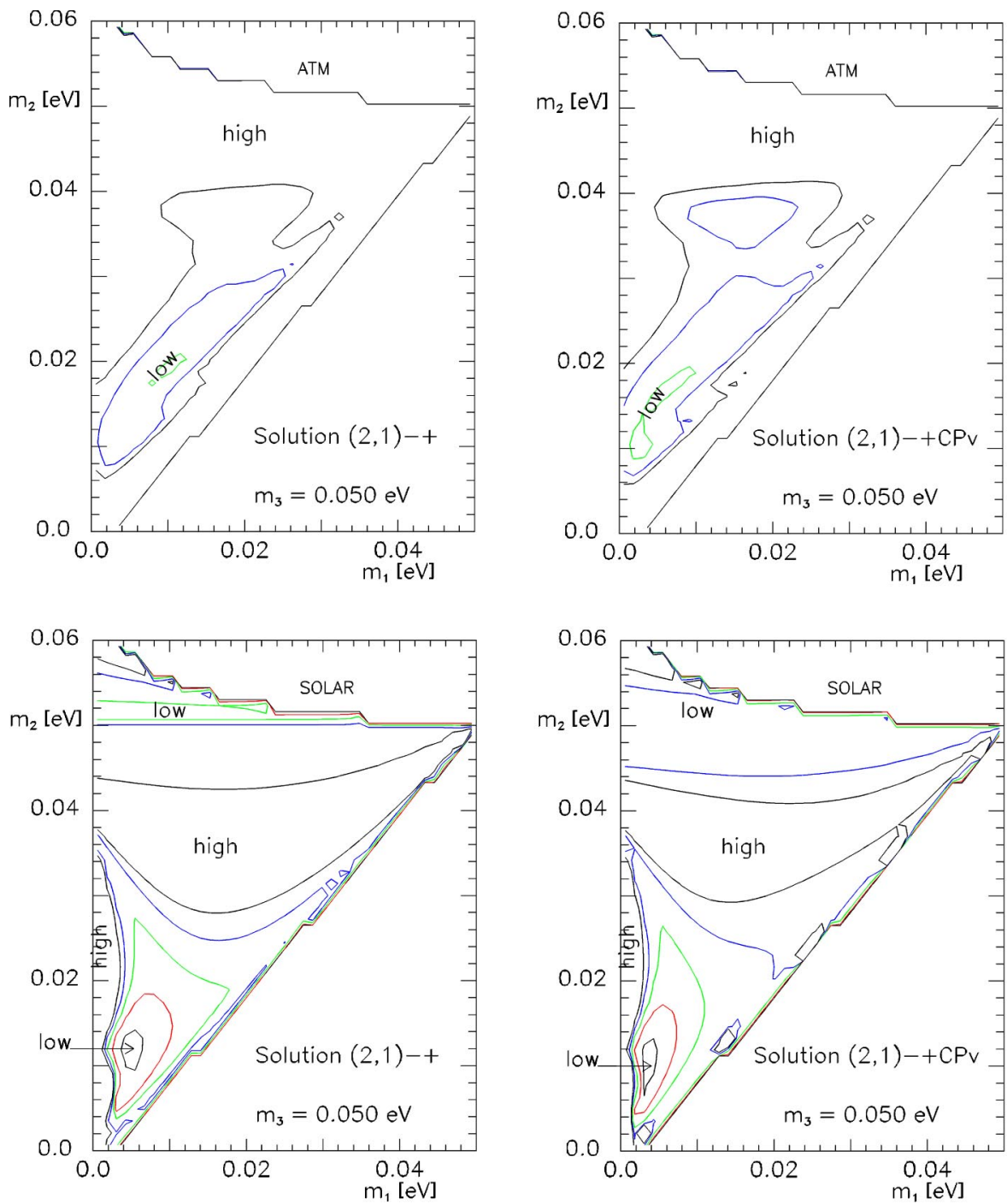

FIG. 5. Fits to the atmospheric and solar neutrino data (procedure A) for the mixing matrix denoted " $(2,1)-+$ " (see Sec. V). Contours are given at $\chi^{2}=5,10,15$, 20, 25. Sum also at $30,35,40,45$, 50. Left panels: $C P$ conservation; right panels: $C P$ nonconservation.

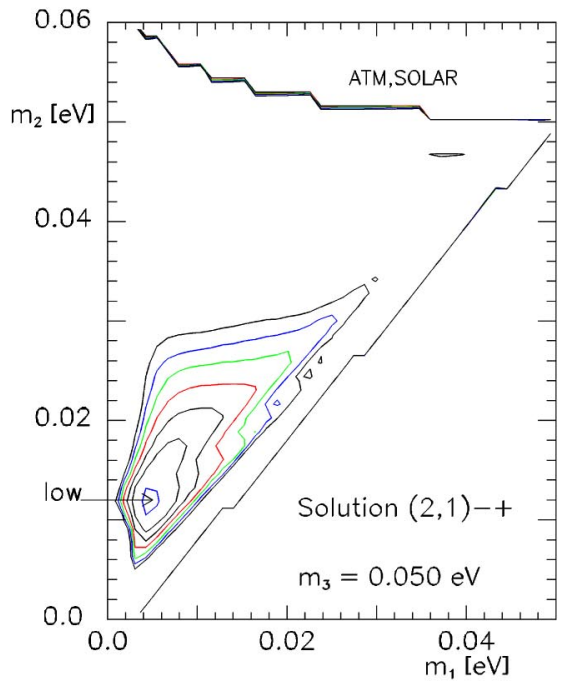

with $\omega_{1}, \omega_{2}, \omega_{3}, \chi_{2}$, and $\chi_{3}$ all real.

We now have to address a small complication due to the possible nonreality of the mass matrix induced by $C P$ nonconservation, and the fact that also the charged lepton states have to be rotated. Consider the case of $C P$ nonconservation,

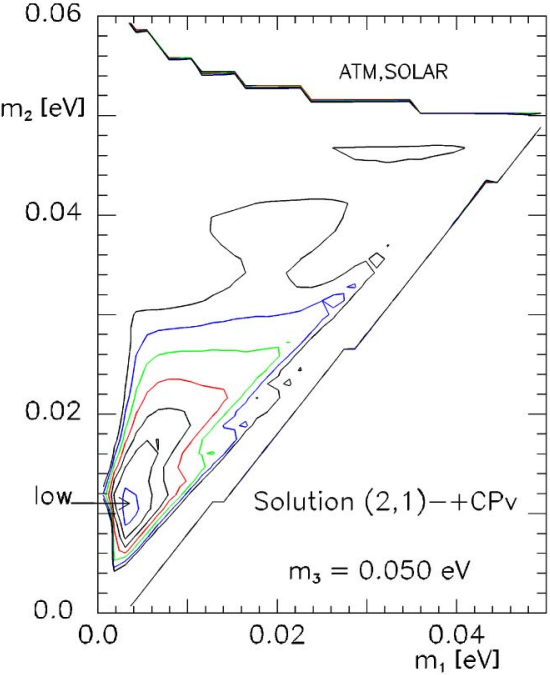

i.e., $\epsilon=i$. In order to follow as closely as possible the procedure of [24], we need to rotate to a neutrino basis which in the absence of matter $[D(r)=0]$ becomes that of the mass eigenstates. This now involves diagonalizing the lower righthand part not of $[M(\nu)]^{2}$, Eq. (7.2), but of 


$$
N^{2}=R^{\mathrm{T}}(\ell) M^{2} R(\ell)=\left[\begin{array}{lll}
\left(N^{2}\right)_{11} & \left(N^{2}\right)_{12} & \left(N^{2}\right)_{13} \\
\left(N^{2}\right)_{12}^{*} & \left(N^{2}\right)_{22} & \left(N^{2}\right)_{23} \\
\left(N^{2}\right)_{13}^{*} & \left(N^{2}\right)_{23}^{*} & \left(N^{2}\right)_{33}
\end{array}\right],
$$

where not only the $(1,2)$ and $(1,3)$ elements are complex [together with $(2,1)$ and $(3,1)]$ [cf. Eq. (7.2)], but also the $(2,3)$ and $(3,2)$ elements. This prevents a simple diagonalization like in [24].

However, we may make the lower right-hand part real by a unitary $2 \times 2$ rotation $\mathcal{U}$. In other words, we perform the transformation

$$
\mathcal{U}\left[\begin{array}{ll}
\left(N^{2}\right)_{22} & \left(N^{2}\right)_{23} \\
\left(N^{2}\right)_{23}^{*} & \left(N^{2}\right)_{33}
\end{array}\right] \mathcal{U}^{\dagger}=\left[\begin{array}{ll}
x & y \\
y & z
\end{array}\right], \quad \text { with }\left[\begin{array}{l}
\bar{\phi}_{2} \\
\bar{\phi}_{3}
\end{array}\right]=\mathcal{U}\left[\begin{array}{l}
\phi_{2} \\
\phi_{3}
\end{array}\right] .
$$

By also defining

$$
\begin{aligned}
& {\left[\begin{array}{cc}
\cos \theta_{0} & -\sin \theta_{0} \\
\sin \theta_{0} & \cos \theta_{0}
\end{array}\right] \frac{r_{0}}{2 p}\left[\begin{array}{ll}
x & y \\
y & z
\end{array}\right]\left[\begin{array}{cc}
\cos \theta_{0} & \sin \theta_{0} \\
-\sin \theta_{0} & \cos \theta_{0}
\end{array}\right]} \\
& \quad=\left[\begin{array}{cc}
\omega_{2} & 0 \\
0 & \omega_{3}
\end{array}\right]
\end{aligned}
$$

together with

$$
\omega_{1}=\frac{r_{0}}{2 p}\left(N^{2}\right)_{11}, \quad\left[\begin{array}{l}
\chi_{2} \\
\chi_{3}
\end{array}\right]=\frac{r_{0}}{2 p}\left[\begin{array}{cc}
\cos \theta_{0} & -\sin \theta_{0} \\
\sin \theta_{0} & \cos \theta_{0}
\end{array}\right]\left[\begin{array}{l}
\left(N^{2}\right)_{12} \\
\left(N^{2}\right)_{13}
\end{array}\right]
$$

and

$$
\psi_{1}(u)=\phi_{1}(u), \quad\left[\begin{array}{l}
\psi_{2}(u) \\
\psi_{3}(u)
\end{array}\right]=\left[\begin{array}{cc}
\cos \theta_{0} & -\sin \theta_{0} \\
\sin \theta_{0} & \cos \theta_{0}
\end{array}\right]\left[\begin{array}{c}
\bar{\phi}_{2}(u) \\
\bar{\phi}_{3}(u)
\end{array}\right],
$$

then Eq. (7.1) can be written in the desired form (7.4). The $\omega$ 's are real, but the $\chi_{2}$ and $\chi_{3}$ will in general be complex. The rotations among $\phi_{2}$ and $\phi_{3}$ given by $\mathcal{U}$ and $\theta_{0}$ need not concern us, since we are here only interested in the electron neutrino, $\nu_{e}$.

When $\omega_{2} \neq \omega_{3}$, these $\psi_{1}, \psi_{2}$, and $\psi_{3}$ can be expressed uniquely in terms of a single $\psi$ :

$$
\begin{aligned}
& \psi_{1}=\left(i \frac{d}{d u}-\omega_{2}\right)\left(i \frac{d}{d u}-\omega_{3}\right) \psi, \\
& \psi_{2}=\chi_{2}\left(i \frac{d}{d u}-\omega_{3}\right) \psi, \\
& \psi_{3}=\chi_{3}\left(i \frac{d}{d u}-\omega_{2}\right) \psi,
\end{aligned}
$$

where $\psi$ satisfies the third-order ordinary differential equation [24]

$$
\begin{gathered}
\left(i \frac{d}{d u}-\mu_{1}\right)\left(i \frac{d}{d u}-\mu_{2}\right)\left(i \frac{d}{d u}-\mu_{3}\right) \psi \\
=e^{-u}\left(i \frac{d}{d u}-\omega_{2}\right)\left(i \frac{d}{d u}-\omega_{3}\right) \psi
\end{gathered}
$$

Here, $\mu_{1}, \mu_{2}$, and $\mu_{3}$ are the eigenvalues of the right-hand matrix in Eq. (7.4) (without the term $e^{-u}$ ), ordered such that

$$
\mu_{1} \leqslant \mu_{2} \leqslant \mu_{3} .
$$

Equation (7.11) is the differential equation for the generalized hypergeometric function ${ }_{2} F_{2}$-see, for example, $\mathrm{p}$. 184 of [25]. Three linearly independent solutions of this third-order differential equation (7.11) are

$$
\begin{aligned}
\psi^{(1)}= & K_{1} e^{-i \mu_{1} u} \\
& \times{ }_{2} F_{2}\left[\begin{array}{rr}
-i\left(\omega_{2}-\mu_{1}\right), & -i\left(\omega_{3}-\mu_{1}\right) \\
1-i\left(\mu_{2}-\mu_{1}\right), & 1-i\left(\mu_{3}-\mu_{1}\right)
\end{array} \mid i e^{-u}\right], \\
\psi^{(2)=} & K_{2} e^{-i \mu_{2} u} \\
& { }_{2} F_{2}\left[\begin{array}{cc}
-i\left(\omega_{2}-\mu_{2}\right), & -i\left(\omega_{3}-\mu_{2}\right) \\
1-i\left(\mu_{1}-\mu_{2}\right), & 1-i\left(\mu_{3}-\mu_{2}\right)
\end{array} \mid i e^{-u}\right], \\
\psi^{(3)}= & K_{3} e^{-i \mu_{3} u} \\
& { }_{2} F_{2}\left[\begin{array}{cc}
-i\left(\omega_{2}-\mu_{3}\right), & -i\left(\omega_{3}-\mu_{3}\right) \\
1-i\left(\mu_{1}-\mu_{3}\right), & 1-i\left(\mu_{2}-\mu_{3}\right)
\end{array} \mid i e^{-u}\right],
\end{aligned}
$$

where $K_{1}, K_{2}$, and $K_{3}$ are arbitrary nonzero constants. Since Eq. (7.11) is linear, the general solution is

$$
\psi=C_{1} \psi^{(1)}+C_{2} \psi^{(2)}+C_{3} \psi^{(3)},
$$

from which the $\psi_{1}, \psi_{2}$, and $\psi_{3}$ can be obtained using Eq. (7.10).

For the case of two flavors, the products in Eq. (7.11) consist of one less term each, and a familiar confluent hypergeometric function ${ }_{1} F_{1}$ (also denoted Whittaker function or parabolic cylinder function) is obtained [26].

These functions are trivial when $u \rightarrow \infty$. In fact, outside the Sun, they can be approximated by the exponential prefactors, since

$$
{ }_{2} F_{2}\left[\begin{array}{ll}
a_{1}, & a_{2} \\
b_{1}, & b_{2}
\end{array} \mid 0\right]=1 \text {. }
$$

In order to impose the boundary conditions that only electron neutrinos are produced in the Sun, we have to determine these functions at large and negative values of $u$. The series expansion is in principle convergent, but it is not practical for large absolute values of both parameters and the argument. One possible way of dealing with these generalized hypergeometric functions has been given in [24]. The procedure used there is as follows. First, $\psi_{1}^{(3)}, \psi_{2}^{(3)}$, and $\psi_{3}^{(3)}$ are evaluated approximately using Barnes' integral representation for ${ }_{2} F_{2}$ [25]. Since we have not managed to apply this 
same procedure to the other $\psi$ 's, they are expressed in terms of another generalized hypergeometric function ${ }_{3} F_{1}$. Since ${ }_{3} F_{1}$ has an integral representation in terms of the usual hypergeometric function ${ }_{2} F_{1}$, these ${ }_{3} F_{1}$ can be evaluated by numerical integration. The choice of the contours of integration has been discussed in detail in [24].

For completeness we give in Appendix B asymptotic formulas for these ${ }_{3} F_{1}$. These asymptotic formulas turn out to be quite useful and in particular are accurate for the region of the minimum $\chi^{2}$, to be discussed below.

Some details on the book-keeping of reconstructing the neutrino wave functions from the ${ }_{2} F_{2}$ and ${ }_{3} F_{1}$ are given in Appendix C.

\section{SOLAR NEUTRINOS}

In order to compare the predictions of the model to data, we form a $\chi^{2}$ by comparing the predictions to the available flux data. For the solar-neutrino flux, we take the values given by the so-called "BP00" solar model [27]. For the solar-neutrino data, we use the total rates from the chlorine experiment [13], the gallium experiments [14,15] (we average the two results), the Super-Kamiokande experiment [8], and the SNO experiment $[16,17]$. We adopt the neutrino energy spectra and detector efficiencies as given by Bahcall et al. [28], and, for Super-Kamiokande and SNO, we also include the neutral-current cross section [7]. We do not consider the day-night effect, since this is consistent with zero [8]. Neither do we consider the electron-recoil spectrum, since this is consistent with being flat [29] (see, however [30]). For the solar flux, we integrate over the spectrum $\Phi_{j}\left(E_{\nu}\right)$, taking into account the detector efficiency $\epsilon\left(E_{\nu}\right)$ :

$$
\Phi=\sum_{j} \int d E_{\nu} \Phi_{j}\left(E_{\nu}\right) \epsilon\left(E_{\nu}\right) P_{\nu}\left(E_{\nu}\right)
$$

In Fig. 5 we show the contributions to $\chi^{2}$ from the solarneutrino data, as functions of $m_{1}$ and $m_{2}$, for $m_{3}$ $=0.05 \mathrm{eV}$. As opposed to the atmospheric-neutrino data, the solar-neutrino data give a minimum $\chi^{2}$ that is well localized in the $m_{1}-m_{2}$ plane, with little dependence on $m_{3}$.

The lower panels in Fig. 5 give the corresponding total $\chi^{2}=\chi_{\mathrm{atm}}^{2}+\chi_{\text {solar }}^{2}$. Since the minimum in $\chi_{\text {solar }}^{2}$ is rather welllocalized ("steep"), the location of the overall minimum (for fixed $m_{3}$ ) is largely determined by the minimum in $\chi_{\text {solar }}^{2}$. However, the extent to which the minimum in $\chi_{\text {atm }}^{2}$ overlaps with that of $\chi_{\text {solar }}^{2}$ determines how good the fit is.

The cases considered here are $U(2,1)-+$, with $C P$ conservation (left) and $C P$ nonconservation (right). Similar scans for the other mixing matrices (other solutions, other parities) yield rather similar results. There is little difference between the cases of $C P$ conservation and $C P$ nonconservation.
TABLE III. Best fits to the atmospheric and solar neutrino data from the fitting procedure A. The number of degrees of freedom is 18; masses are in $\mathrm{eV}$.

\begin{tabular}{lccccccc}
\hline \hline Solution & $m_{1}$ & $m_{2}$ & $m_{3}$ & $\chi_{\text {atm }}^{2}$ & $\chi_{\text {solar }}^{2}$ & $\chi^{2}$ & \\
\hline$(1,1)-+$ & 0.0049 & 0.013 & 0.038 & 19.2 & 3.8 & 23.0 & $C P$ cons. \\
same & 0.0036 & 0.012 & 0.037 & 18.0 & 3.7 & 21.7 & $C P$ noncons. \\
$(1,1)--$ & 0.0027 & 0.011 & 0.038 & 17.1 & 3.7 & 20.8 & $C P$ cons. \\
same & 0.0036 & 0.012 & 0.038 & 17.7 & 3.7 & 21.4 & $C P$ noncons. \\
$(1,2)++$ & 0.0047 & 0.008 & 0.052 & 14.9 & 5.8 & 20.7 & $C P$ cons. \\
same & 0.0030 & 0.007 & 0.052 & 15.3 & 5.7 & 21.0 & $C P$ noncons. \\
$(1,2)+-$ & 0.0022 & 0.006 & 0.052 & 16.5 & 6.1 & 22.6 & $C P$ cons. \\
same & 0.0030 & 0.007 & 0.052 & 16.3 & 5.9 & 22.1 & $C P$ noncons. \\
$(2,1)++$ & 0.0045 & 0.008 & 0.052 & 15.1 & 6.1 & 21.2 & $C P$ cons. \\
same & 0.0028 & 0.006 & 0.052 & 15.4 & 5.3 & 20.8 & $C P$ noncons. \\
$(2,1)+-$ & 0.0021 & 0.006 & 0.052 & 16.7 & 6.0 & 22.7 & $C P$ cons. \\
same & 0.0028 & 0.007 & 0.052 & 16.6 & 5.3 & 21.9 & $C P$ noncons. \\
same & 0.0029 & 0.007 & 0.095 & 16.3 & 4.5 & 20.8 & $C P$ noncons. \\
$(2,1)-+$ & 0.0045 & 0.013 & 0.052 & 14.5 & 3.6 & 18.1 & $C P$ cons. \\
same & 0.0035 & 0.011 & 0.053 & 13.8 & 3.6 & 17.3 & $C P$ noncons. \\
$(2,1)--$ & 0.0026 & 0.010 & 0.052 & 12.5 & 3.8 & 16.3 & $C P$ cons. \\
same & 0.0034 & 0.011 & 0.052 & 13.1 & 3.7 & 16.8 & $C P$ noncons. \\
\hline \hline
\end{tabular}

\section{RESULTS}

\section{A. Combining atmospheric and solar data}

Starting at the minima found from two-dimensional scans as described in Secs. VI and VIII, but now with $m_{1}, m_{2}$, and $m_{3}$ all free, we find total minima for a given solution of the mixing matrix. The fitting has been performed using two different procedures. Procedure A is the method proposed by Hata and Langacker [31], where we allow for correlations between different data. Procedure B is a more transparent approach, where the data are treated as uncorrelated, and the SNO data are left out. There are 16 degrees of freedom. The detailed numerical results differ somewhat between these two procedures.

The best such results from fitting procedure A are collected in Table III. Two cases stand out. They are solution " $(2,1)--$ " and " $(2,1)-+$," with $\chi^{2}$ values ranging from 16.3 to 18.1. Thus, solution 2 is favored for the neutrino mass matrix, whereas solution 1 is favored for the charged lepton mass matrix. Also, negative " $b$ parity" is favored, whereas there is no clear preference for a particular " $d$ parity." The latter observation is commented on in Sec. X.

TABLE IV. Best fits to the atmospheric and solar neutrino data from the fitting procedure $\mathrm{B}$. The number of degrees of freedom is 16; masses are in $\mathrm{eV}$.

\begin{tabular}{lccccccc}
\hline \hline Solution & $m_{1}$ & $m_{2}$ & $m_{3}$ & $\chi_{\text {atm }}^{2}$ & $\chi_{\text {solar }}^{2}$ & $\chi^{2}$ & \\
\hline$(2,1)-+$ & 0.0047 & 0.011 & 0.054 & 16.2 & 0.8 & 17.0 & $C P$ cons. \\
same & 0.0034 & 0.010 & 0.054 & 15.0 & 0.8 & 15.8 & $C P$ noncons. \\
$(2,1)--$ & 0.0025 & 0.010 & 0.054 & 15.0 & 0.8 & 15.8 & $C P$ cons. \\
same & 0.0035 & 0.010 & 0.054 & 15.3 & 0.8 & 16.1 & $C P$ noncons. \\
\hline \hline
\end{tabular}


TABLE V. Best fits from the fitting procedure A, including the CHOOZ data [34]. Masses are in eV.

\begin{tabular}{lcccccccc}
\hline \hline Solution & $m_{1}$ & $m_{2}$ & $m_{3}$ & $\chi_{\text {atm }}^{2}$ & $\chi_{\text {solar }}^{2}$ & $\chi_{\text {CHOOZ }}^{2}$ & $\chi^{2}$ & \\
\hline$(2,1)-+$ & 0.0044 & 0.013 & 0.052 & 14.5 & 3.6 & 2.9 & 21.0 & $C P$ cons. \\
same & 0.0034 & 0.011 & 0.052 & 13.6 & 3.7 & 3.1 & 20.4 & $C P$ noncons. \\
$(2,1)--$ & 0.0026 & 0.010 & 0.053 & 12.6 & 3.8 & 3.9 & 20.2 & $C P$ cons. \\
same & 0.0034 & 0.011 & 0.052 & 13.1 & 3.6 & 3.1 & 19.9 & $C P$ noncons. \\
\hline \hline
\end{tabular}

With fitting procedure $\mathrm{B}$, these two solutions have $\chi^{2}$ ranging from 15.8 to 17.0 as can be seen from Table IV.

There is a strong clustering of $m_{3}$ values at $0.052-0.054 \mathrm{eV}$, for different solutions and with different "parities." However, there are also a few fits which are not much inferior with $m_{3}$ values around $0.04 \mathrm{eV}$, and one at $0.095 \mathrm{eV}$. These have a $\chi^{2}$ that is higher by about 4 units.

The best-fit mass values may roughly be related to the conventional $[32,33]$ atmospheric and solar neutrino parameters as $\Delta m_{\mathrm{atm}}^{2} \simeq m_{3}^{2}-m_{2}^{2}=2.6 \times 10^{-3} \mathrm{eV}^{2}$ and $\Delta m_{\text {solar }}^{2}$ $\simeq m_{2}^{2}-m_{1}^{2}=(0.9-1.5) \times 10^{-4} \mathrm{eV}^{2}$.

\section{B. Impact of $\mathrm{CHOOZ}$ data}

The CHOOZ data [34] are known to disfavor models with "large" values of $\left|U_{e 3}\right|$. In our model, large $\left|U_{e 3}\right|$ typically require $m_{2}$ comparable with $m_{3}$, as can be seen in the example shown in Fig. 4. Thus, we do not expect the minima shown in Table III to be significantly altered by the inclusion of the CHOOZ data. This is in fact the case, as shown in Table V. For the fits of Table III, the inclusion of the CHOOZ data (14 data points) increases the total $\chi^{2}$ by $3-4$ units, without changing the best-fit mass values.

We show in Table VI the relevant mixing elements corresponding to these best fits. Since these cases all refer to solution 1 for the charged leptons, then to a good approximation we have $U \approx R(\nu)$. Hence, the cases presented here are pairwise related $(d \rightarrow-d)$ by the symmetry of $R(\nu)$, discussed in Sec. IV.
In the decoupling approximation (neglecting $U_{e 3}$, which ranges from 0.03 to 0.13 ), the relevant quantities for solar and atmospheric neutrinos are $\left|U_{e 1} U_{e 2}\right| \simeq 0.42-0.44$ and $\left|U_{\mu 3} U_{\tau 3}\right| \simeq 0.39-0.40$, respectively, corresponding to large mixing in both cases. As shown in Table VI, when $C P$ is not conserved, the amount is given by

$$
J_{C P}= \pm 6.0 \times 10^{-3}
$$

for neutrinos. This is much larger than the corresponding quantity for quarks as given by Eq. (2.9).

\section{DISCUSSION}

Our theoretical description of neutrino oscillations suffers from the fact that the calculation of the three-flavor MSW effect relies on a somewhat crude solar model electron density. Nevertheless, it is most rewarding to find that the determination of the three neutrino masses can be carried out successfully, i.e., in very reasonable agreement with the experimental data. We have found excellent fits to the data with $m_{3}=(52-54) \times 10^{-3} \mathrm{eV}, m_{2}=(10-13) \times 10^{-3} \mathrm{eV}$, and $m_{1}$ $=(2-5) \times 10^{-3} \mathrm{eV}$.

In a regime where

$$
m_{1} \ll m_{2} \ll m_{3},
$$

the atmospheric neutrino data determine $m_{3}=\mathcal{O}\left(\sqrt{\Delta m_{\mathrm{atm}}^{2}}\right)$ $\simeq 0.05 \mathrm{eV}$, and the solar neutrino data determine $m_{2}$

TABLE VI. Mixing matrix elements $U_{\alpha j}(\alpha=e, \mu, \tau)$ for the best fits, given as (modulus, phase $/ \pi$ ).

\begin{tabular}{|c|c|c|c|c|c|c|c|}
\hline Solution & $m_{1}$ & $m_{2}$ & $m_{3}$ & $U_{\alpha 1}$ & $U_{\alpha 2}$ & $U_{\alpha 3}$ & $J_{C P}$ \\
\hline$(2,1)-+$ & 0.0044 & 0.013 & 0.052 & $\begin{array}{l}(0.87,0.00) \\
(0.39,0.00) \\
(0.31,1.00)\end{array}$ & $\begin{array}{l}(0.48,1.00) \\
(0.81,0.00) \\
(0.33,1.00)\end{array}$ & $\begin{array}{l}(0.13,0.00) \\
(0.44,0.00) \\
(0.89,0.00)\end{array}$ & 0.00 \\
\hline$(2,1)-+$ & 0.0034 & 0.011 & 0.052 & $\begin{array}{c}(0.86,-0.49) \\
(0.43,0.04) \\
(0.28,1.00)\end{array}$ & $\begin{array}{c}(0.50,0.47) \\
(0.79,-0.01) \\
(0.34,1.00)\end{array}$ & $\begin{array}{c}(0.08,-0.38) \\
(0.44,0.00) \\
(0.90,0.00)\end{array}$ & 0.0060 \\
\hline$(2,1)--$ & 0.0026 & 0.010 & 0.053 & $\begin{array}{l}(0.85,0.00) \\
(0.46,1.00) \\
(0.26,0.00)\end{array}$ & $\begin{array}{l}(0.52,0.00) \\
(0.77,0.00) \\
(0.36,1.00)\end{array}$ & $\begin{array}{l}(0.03,1.00) \\
(0.44,0.00) \\
(0.90,0.00)\end{array}$ & 0.00 \\
\hline$(2,1)--$ & 0.0034 & 0.011 & 0.052 & $\begin{array}{c}(0.86,-0.51) \\
(0.43,0.96) \\
(0.28,0.00)\end{array}$ & $\begin{array}{c}(0.51,-0.47) \\
(0.79,0.01) \\
(0.34,1.00)\end{array}$ & $\begin{array}{l}(0.08,0.38) \\
(0.43,0.00) \\
(0.90,0.00)\end{array}$ & -0.0060 \\
\hline
\end{tabular}
Masses are in $\mathrm{eV}$. 
$=\mathcal{O}\left(\sqrt{\Delta m_{\text {solar }}^{2}}\right) \simeq 0.01 \mathrm{eV}$. The test of the model then lies in (i) reproducing the hierarchy (10.1) and (ii) the determination of $m_{1}$.

The fact that large mixing is required by the atmospheric as well as the solar neutrino data essentially forces the model into a region of parameter space where there is a strong hierarchy. To some extent, this can be read off from Figs. $2-4$.

Another issue is to what extent the data can distinguish the different discrete parameters of the model, solution 1 vs solution 2, as well as the $b$ and $d$ "parities." The data favor solution 2 for the neutrino mixing and solution 1 for the charged leptons, both by a margin of 4.4 units of $\chi^{2}$.

The best fits have been found for charged lepton solution 1. Since the charged lepton masses are strongly hierarchical, the rotation matrix corresponding to solution 1 is very close to the unit matrix. Thus, the overall neutrino mixing matrix $U$ is rather close to $R(\nu)$. This explains why the mass values obtained are close to those presented earlier [18].

It is of some interest to compare in more detail with the case

$$
U=R(\nu)
$$

For the two best fits, solutions $(2)-+$ and $(2)--$, we find $\chi^{2}=17.2$ and 16.7 , respectively, with $m_{3}=0.057$ and $0.052 \mathrm{eV}$. The former solution is the best fit of [18].

Actually, in the limit of no mixing in the charged-lepton sector, $R(\ell)=1$, when $U=R(\nu)$, we see that the atmospheric transition probability (6.3) is invariant under $b \rightarrow-b$, as well as under $d \rightarrow-d$ or $d \rightarrow i d$. Similarly, for the MSW equation (7.2), the sign change $b \rightarrow-b$ can be compensated for by a wave function sign change, $\phi_{3} \rightarrow-\phi_{3}$, and the sign change $d \rightarrow-d$ (or $d \rightarrow i d$ ) can be compensated for by sign (or phase) changes of $\phi_{2}$ and $\phi_{3}$. Thus, these are exact symmetries of $\left|\phi_{1}\right|^{2}$ in this limit of $R(\ell)=1$. They are therefore approximate symmetries for solution 1 for the charged-lepton sector.

It is also interesting to compare with the rather different model proposed by [35]. The masses they find are $m_{3}$ $=0.0506 \mathrm{eV}, \quad m_{2}=(7.46-7.48) \times 10^{-3} \mathrm{eV}, \quad$ and $m_{1}$ $=(2.39-2.43) \times 10^{-3} \mathrm{eV}$. As mentioned above, the fact that $m_{3}$ and $m_{2}$ are rather similar to the values we find is unavoidable within a hierarchical fit.

We add a comment on $C P$ nonconservation in the lepton sector. In the work of Lehmann et al. [1], given the other parameters, $C P$ nonconservation is maximal. For this reason, when $\epsilon=i$, the lepton $C P$ nonconservation is also maximal in the same sense. In other words, the lepton $C P$ nonconservation is either zero $(\epsilon=1)$ or maximal $(\epsilon=i)$. Thus there are only two cases instead of a continuum.

The absolute values of the elements of the mixing matrices are somewhat different in these two cases. These differences are not sufficiently large to decide experimentally whether $C P$ is conserved or not in the lepton sector, since both give comparable fits, as shown in Table V. Note that the $\chi^{2}$ is slightly smaller (by $0.3-0.6$ ) for the case of $C P$ nonconservation. That the Jarlskog determinant (9.1) for leptons is significantly larger than that of Eq. (2.9) for quarks is related to the fact that the ratios of neutrino masses obtained here for leptons are larger than those for quarks (for a more general discussion, see [36]).

\section{ACKNOWLEDGMENTS}

It is a pleasure to thank Geir Vigdel for very useful discussions. One of us (T.T.W.) wishes to thank the Theory Division of CERN for its kind hospitality. This work was supported in part by the Fund for Scientific Research, Flanders-Belgium and by the Interuniversity Attraction Poles, Belgium (P5-11-35); in part by the Research Council of Norway; and in part by the United States Department of Energy under Grant No. DE-FG02-84ER40158.

\section{APPENDIX A}

We discuss in this appendix some elementary properties of the mass matrix of Ref. [1]:

$$
M=\left(\begin{array}{ccc}
0 & i d & 0 \\
-i d & c & b \\
0 & b & a
\end{array}\right),
$$

with $b^{2}=8 c^{2}$. From Eq. (3.5), this $M$ is diagonalized by

$$
M=\operatorname{diag}(i, 1,1) R \operatorname{diag}\left(m_{1},-m_{2}, m_{3}\right) R^{\mathrm{T}} \operatorname{diag}(-i, 1,1) .
$$

The rotation matrix $R$ is discussed in Sec. IV. The case of the mass matrix without the factors of $i$ and $-i$ is entirely similar. The relations between the elements of $M$ and the masses have been given in Eq. (2.7).

Consider first the triangular region (2.8) studied in Ref. [1]. In this region, the $S_{1}, S_{2}$, and $S_{3}$ of Eq. (2.7) satisfy

$$
S_{3}-S_{1} S_{2}=\left(m_{2}-m_{1}\right)\left(m_{3}-m_{2}\right)\left(m_{3}+m_{1}\right)>0 \text {. }
$$

Also from Eq. (2.7), the 33 entry of $M$, namely $a$, satisfies the cubic equation (3.11), and the possible signs of $a$ have been discussed immediately thereafter. Since $c=S_{1}-a$, the corresponding cubic equation for $c$ is

$$
9 c^{3}-10 S_{1} c^{2}+\left(S_{1}^{2}+S_{2}\right) c+\left(S_{3}-S_{1} S_{2}\right)=0 .
$$

When Eq. (3.11) has three real solutions, so does Eq. (A4). Furthermore, by the inequality (A3), two of the real solutions must be positive, while the third one is negative. A comparison with what is known about Eq. (3.11) shows that, in the triangular region (2.8),

$$
\begin{aligned}
& c<0 \text { for solution 1, } \\
& c>0 \text { for solution } 2 .
\end{aligned}
$$

When $m_{2} \rightarrow m_{1}$ or $m_{3} \rightarrow m_{2}$, it follows from Eq. (A3) that $S_{3}-S_{1} S_{2}=0$. In either limit, Eq. (A4) becomes a quadratic equation when $c \neq 0$ :

$$
9 c^{2}-10 S_{1} c+\left(S_{1}^{2}+S_{2}\right)=0 .
$$


In the limit $m_{2} \rightarrow m_{1}$, this has two positive roots. In the limit $m_{2} \rightarrow m_{3}$, this has one positive and one negative root. Therefore, for solution $1, c$ remains negative when $m_{2} \rightarrow m_{1}$ or $m_{2} \rightarrow m_{3}$. On the other hand, for solution 2, $c$ remains positive when $m_{2} \rightarrow m_{1}$, whereas

$$
c \rightarrow 0
$$

when $m_{2} \rightarrow m_{3}$.

From the discussion after Eq. (3.11), the interesting case is where this cubic equation has three real roots. This holds not only in the triangular region (2.8) but in a larger region. The boundary of this larger region is given by the straight line $m_{1}=0$ together with curves obtained by setting the discriminant of the cubic equation (3.11) to zero:

$$
\begin{aligned}
& \left(8 S_{1}^{2}+S_{2}\right)^{2}\left(S_{1}^{2}-36 S_{2}\right)-2187 S_{3}^{2}+2380 S_{1}^{3} S_{3} \\
& \quad+2754 S_{1} S_{2} S_{3}=0 .
\end{aligned}
$$

The resulting region is only slightly larger than the triangle (2.8), and has been discussed in Sec. III. Let the region shown in Fig. 1 be called $\mathcal{R}$, while $\mathcal{R}_{0}$ denotes the triangle (2.8); then $\mathcal{R}$ consists of $\mathcal{R}_{0}$ together with $\mathcal{R}_{1}$ (the small region where $m_{1}>m_{2}$ ) and $\mathcal{R}_{2}$ (the region where $m_{2}>m_{3}$ ). The extension of Eq. (A5) to $\mathcal{R}_{1}$ and $\mathcal{R}_{2}$ gives simply

$$
\begin{aligned}
& c<0 \text { for solution } 1 \text { in all of } \mathcal{R} \\
& c>0 \text { for solution } 2 \text { in } \quad \mathcal{R}_{0} \text { and } \mathcal{R}_{1} \\
& c<0 \text { for solution } 2 \text { in } \quad \mathcal{R}_{2} .
\end{aligned}
$$

The next property to be discussed is the behavior of the $m$ 's when both $c$ and $d$ are small compared with $a$ :

$$
|c| \ll a \quad \text { and } \quad|d| \ll a,
$$

the relative magnitude of $c$ and $d$ being arbitrary. In general, by Eq. (2.7), the masses $m_{1},-m_{2}$, and $m_{3}$ satisfy the cubic equation

$$
\lambda^{3}-(a+c) \lambda^{2}+\left(a c-8 c^{2}-d^{2}\right) \lambda+a d^{2}=0 .
$$

In the limiting case (A10), one of the solutions is

$$
\lambda=m_{3} \sim a
$$

while the other two solutions are both small. These two small solutions, $m_{1}$ and $-m_{2}$, are determined approximately by the quadratic equation

$$
-a \lambda^{2}+\left(a c-d^{2}\right) \lambda+a d^{2}=0
$$

The solutions of Eq. (A13) are

$$
\begin{aligned}
\lambda & =\frac{1}{2 a}\left[a c-d^{2} \pm \sqrt{\left(a c-d^{2}\right)^{2}+4 a^{2} d^{2}}\right] \\
& \sim \frac{1}{2}\left[c \pm \sqrt{c^{2}+4 d^{2}}\right],
\end{aligned}
$$

or

$$
\begin{aligned}
& m_{1}=\frac{1}{2}\left[c+\sqrt{c^{2}+4 d^{2}}\right], \\
& m_{2}=\frac{1}{2}\left[-c+\sqrt{c^{2}+4 d^{2}}\right] .
\end{aligned}
$$

Note that these approximate solutions are applicable only to solution 1, because Eq. (3.13) implies that, for solution 2, $c$ cannot satisfy the inequality (A10). Equation (A15) shows directly that $c<0$ for solution 1 in the triangular region (2.8).

\section{APPENDIX B}

For completeness we give here asymptotic formulas for the ${ }_{3} F_{1}$ required for reconstructing the ${ }_{2} F_{2}$. These asymptotic formulas turn out to be quite useful and in particular are accurate for the regions of the fits discussed in Sec. VIII. For the wave functions of the electron neutrino, $\psi_{1}$ of Eq. (7.4), the two relevant ${ }_{3} F_{1}$ are (cf. [24])

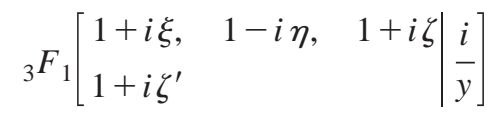

$$
\begin{aligned}
& =\frac{1}{(2 \pi)^{2}}\left(e^{i \pi / 2} y\right)^{1+i \xi} \\
& \times e^{\pi \zeta^{\prime}} \frac{\Gamma\left(1+i \zeta^{\prime}\right) \Gamma(i \eta) \Gamma\left(1-i \eta-i \zeta^{\prime}\right)}{\Gamma(1+i \xi)} I,
\end{aligned}
$$

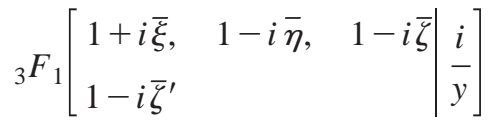

$$
\begin{aligned}
& =\left(e^{i \pi / 2} y\right)^{1+i \bar{\xi}} \frac{\Gamma\left(1-i \zeta^{\prime}\right)}{\Gamma(1+i \bar{\xi}) \Gamma(1-i \bar{\eta}) \Gamma\left(i \bar{\eta}-i \zeta^{\prime}\right)} \bar{I},
\end{aligned}
$$

where

$$
\begin{aligned}
I= & \int_{0}^{\infty} d t \int_{\mathcal{P}} d s(1+s+s t)^{-1} \exp \{-i[t y-\xi \ln t+\eta \ln s \\
& \left.\left.-\left(\zeta-\zeta^{\prime}\right) \ln (1+s)+\zeta \ln (1+s+s t)\right]\right\}, \\
\bar{I}= & \int_{0}^{\infty} d t \int_{0}^{\infty} d s(1+s+s t)^{-1} \exp \{-i[t y-\bar{\xi} \ln t+\bar{\eta} \ln s \\
& \left.\left.+\left(\bar{\zeta}-\zeta^{\prime}\right) \ln (1+s)-\bar{\zeta} \ln (1+s+s t)\right]\right\}, \\
\xi= & \mu_{2}-\omega_{2}, \quad \eta=\omega_{2}-\mu_{1}, \quad \zeta=\mu_{3}-\omega_{2}, \\
\bar{\xi}= & \mu_{3}-\omega_{3}, \quad \bar{\eta}=\omega_{3}-\mu_{2}, \quad \bar{\zeta}=\omega_{3}-\mu_{1},
\end{aligned}
$$

and 


$$
y=e^{-u} .
$$

Note that all these quantities in Eq. (B5) are positive because

$$
\mu_{1}<\omega_{2}<\mu_{2}<\omega_{3}<\mu_{3} \text {. }
$$

The double integrals for $I$ and $\bar{I}$ of Eqs. (B3) and (B4) can be carried out approximately using the method of stationary phase, and the resulting asymptotic formulas are

$$
\begin{aligned}
I \sim & 2 \pi \sigma_{0}^{-1 / 2}\left[-\frac{\xi\left(\zeta-\zeta^{\prime}\right)\left(\sigma_{0}-1-t_{0}\right)^{2}}{\left(\sigma_{0}-1\right)^{2} t_{0}^{2}}+\frac{\zeta\left(\zeta-\zeta^{\prime}\right)}{\left(\sigma_{0}-1\right)^{2}}\right. \\
& \left.+\frac{\left(1+t_{0}\right) \xi \zeta}{t_{0}^{2}}+\frac{\zeta^{2}}{\sigma_{0}-1-t_{0}}\right]^{-1 / 2} \exp \left\{-i\left[t_{0} y-\xi \ln t_{0}\right.\right. \\
& -\left(\eta+\zeta^{\prime}\right) \ln \sigma_{0}-\left(\zeta-\zeta^{\prime}\right) \ln \left(\sigma_{0}-1\right) \\
& \left.\left.+\zeta \ln \left(\sigma_{0}-1-t_{0}\right)\right]\right\}
\end{aligned}
$$

and

$$
\begin{aligned}
\bar{I} \sim 2 & \pi \bar{\sigma}_{0}^{-1 / 2}\left[-\frac{\bar{\xi}\left(\bar{\zeta}-\zeta^{\prime}\right)\left(\bar{\sigma}_{0}+1+\bar{t}_{0}\right)^{2}}{\left(\bar{\sigma}_{0}+1\right)^{2} \bar{t}_{0}^{2}}-\frac{\bar{\zeta}\left(\bar{\zeta}-\zeta^{\prime}\right)}{\left(\bar{\sigma}_{0}+1\right)^{2}}\right. \\
& \left.+\frac{\left(1+\bar{t}_{0}\right) \bar{\xi} \bar{\zeta}}{\bar{t}_{0}^{2}}+\frac{\bar{\zeta}^{2}}{\bar{\sigma}_{0}+1+\bar{t}_{0}}\right]^{-1 / 2} \\
& \times \exp \left\{-i\left[\bar{t}_{0} y-\bar{\xi} \ln \bar{t}_{0}-\left(\bar{\eta}-\zeta^{\prime}\right) \ln \bar{\sigma}_{0}\right.\right. \\
& \left.\left.+\left(\bar{\zeta}-\zeta^{\prime}\right) \ln \left(\bar{\sigma}_{0}+1\right)-\bar{\zeta} \ln \left(\bar{\sigma}_{0}+1+\bar{t}_{0}\right)\right]\right\}
\end{aligned}
$$

where $\sigma_{0}$ and $t_{0}$ are determined by

$$
\begin{gathered}
y-\frac{\xi}{t_{0}}-\frac{\zeta}{\sigma_{0}-1-t_{0}}=0, \\
\eta+\frac{\zeta-\zeta^{\prime}}{\sigma_{0}-1}-\frac{\left(1+t_{0}\right) \zeta}{\sigma_{0}-1-t_{0}}=0,
\end{gathered}
$$

while $\bar{\sigma}_{0}$ and $\bar{t}_{0}$ are determined by

$$
\begin{gathered}
y-\frac{\bar{\xi}}{\bar{t}_{0}}-\frac{\bar{\zeta}}{\bar{\sigma}_{0}+1+\bar{t}_{0}}=0, \\
\bar{\eta}+\frac{\bar{\zeta}-\zeta^{\prime}}{\bar{\sigma}_{0}+1}-\frac{\left(1+\bar{t}_{0}\right) \bar{\zeta}}{\bar{\sigma}_{0}+1+\bar{t}_{0}}=0 .
\end{gathered}
$$

Note that the $\sigma_{0}$ here is equal to $-1 / s_{0}$, where $s_{0}$ is defined in the Appendix of [24].

\section{APPENDIX C}

We shall provide here some further details, beyond what was given in [24], on the reconstruction of $\psi^{(1)}$ and $\psi^{(2)}$ from the ${ }_{3} F_{1}$ 's.

We introduce subscripts $i$ and $k$ to label the cases (5.3)(5.5) and (5.12), (5.13), respectively, of [24]. Then, Eqs.
(5.12), (5.13) of [24] may be rewritten as

$$
\hat{f}_{i}^{(k)}(z)=G_{i k}\left[Y_{i k 1} f_{i}^{(1)}(z)+Y_{i k 2} f_{i}^{(2)}(z)+Y_{i k 3} f_{i}^{(3)}(z)\right]
$$

where

$$
G_{i 1}=\frac{\pi \Gamma\left(1-\alpha_{2}+\alpha_{1}\right)}{\Gamma\left(-\beta_{1}+\alpha_{1}\right) \Gamma\left(-\beta_{2}+\alpha_{1}\right) \Gamma\left(-\beta_{3}+\alpha_{1}\right)}
$$

and

$$
Y_{i 11}=\frac{\Gamma\left(-\beta_{2}+\beta_{1}\right) \Gamma\left(-\beta_{3}+\beta_{1}\right)}{\Gamma\left(1-\alpha_{1}+\beta_{1}\right) \Gamma\left(1-\alpha_{2}+\beta_{1}\right)} \frac{1}{\sin \pi\left(\alpha_{1}-\beta_{1}\right)},
$$

etc., with $\alpha_{i}$ and $\beta_{j}$ given by Eqs. (5.2)-(5.5) of [24]. Furthermore,

$$
\left\{G_{i 2}, Y_{i 2 j}\right\}=\left\{G_{i 1}, Y_{i 1 j}\right\}_{\alpha_{1} \leftrightarrow \alpha_{2}} .
$$

Note that $Y_{i 2 j}=Y_{i 1 j}$ apart from the change of argument of $\sin \left[\pi\left(\alpha_{k}-\beta_{j}\right)\right]$.

Define now

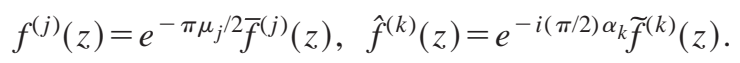

Then, Eq. (C1) can be rewritten as

$$
\widetilde{f}_{i}^{(k)}(z)=B_{i k 1} \bar{f}_{i}^{(1)}(z)+B_{i k 2} \bar{f}_{i}^{(2)}(z)+B_{i k 3} \bar{f}_{i}^{(3)}(z)
$$

with

$$
B_{i k j}=G_{i k} Y_{i k j} e^{(\pi / 2)\left[i \alpha_{k}-\mu_{j}\right]} .
$$

For each $i$, we have the following two equations:

$$
\begin{aligned}
& B_{i 11} \bar{f}_{i}^{(1)}(z)+B_{i 12} \bar{f}_{i}^{(2)}(z)=\left[\widetilde{f}_{i}^{(1)}(z)-B_{i 13} \bar{f}_{i}^{(3)}(z)\right], \\
& B_{i 21} \bar{f}_{i}^{(1)}(z)+B_{i 22} \bar{f}_{i}^{(2)}(z)=\left[\widetilde{f}_{i}^{(2)}(z)-B_{i 23} \bar{f}_{i}^{(3)}(z)\right] .
\end{aligned}
$$

Here, $\tilde{f}_{i}^{(1)}(z)$ and $\widetilde{f}_{i}^{(2)}(z)$ are given in terms of ${ }_{3} F_{1}$ 's, whereas the $\bar{f}_{i}^{(3)}(z)$ are given in terms of ${ }_{2} F_{2}$ 's. These are then solved for $\bar{f}_{i}^{(1)}(z)$ and $\bar{f}_{i}^{(2)}(z)$, from which the ${ }_{2} F_{2}$ of Eq. (5.7) of [24] are obtained.

To obtain the physical neutrino wave functions, one has to rotate back to the $\phi_{i}$ of Eq. (7.1). 
[1] H. Lehmann, C. Newton, and T. T. Wu, Phys. Lett. B 384, 249 (1996).

[2] N. Cabibbo, Phys. Rev. Lett. 10, 531 (1963); M. Kobayashi and T. Maskawa, Prog. Theor. Phys. 49, 652 (1973).

[3] H. Harari, H. Haut, and J. Weyers, Phys. Lett. 78B, 459 (1978); J.-M. Frère, ibid. 80B, 369 (1979); P. Kaus and S. Meshkov, Mod. Phys. Lett. A 3, 1251 (1988); 4, 603(E) (1989); M. Tanimoto, Phys. Rev. D 41, 1586 (1990); M. Fukugita, M. Tanimoto, and T. Yanagida, Prog. Theor. Phys. 89, 263 (1993); Phys. Rev. D 57, 4429 (1998); Y. Nomura and T. Yanagida, ibid. 59, 017303 (1999).

[4] H. Fritzsch and Z. Z. Xing, Phys. Lett. B 372, 265 (1996); Phys. Rev. D 57, 594 (1998).

[5] G. Altarelli and F. Feruglio, Phys. Rep. 320, 295 (1999); hep$\mathrm{ph} / 0206077$.

[6] R. G. Roberts, A. Romanino, G. G. Ross, and L. VelascoSevilla, Nucl. Phys. B615, 358 (2001).

[7] Super-Kamiokande Collaboration, Y. Fukuda et al., Phys. Lett. B 433, 9 (1998); 436, 33 (1998); Phys. Rev. Lett. 81, 1562 (1998).

[8] Super-Kamiokande Collaboration, Y. Fukuda et al., Phys. Rev. Lett. 81, 1158 (1998); 81, 4279(E) (1998); Super-Kamiokande Collaboration, S. Fukuda et al., ibid. 86, 5651 (2001).

[9] S. L. Glashow, Nucl. Phys. 22, 579 (1961); S. Weinberg, Phys. Rev. Lett. 19, 1264 (1967); A. Salam, in Elementary Particle Theory: Relativistic Groups and Analyticity (Nobel Symposium No. 8), edited by N. Svartholm (Almqvist and Wiksell, Stockholm, 1968), p. 367; S. L. Glashow, J. Iliopoulos, and L. Maiani, Phys. Rev. D 2, 1285 (1970).

[10] For recent reviews, see E. K. Akhmedov, Lectures given at ICTP Summer School in Particle Physics, Trieste, Italy, 1999, hep-ph/0001264; W. C. Haxton, in Current Aspects of Neutrino Physics, edited by David Caldwell (Springer-Verlag, Berlin, 2001), p. 65, nucl-th/0004052; M. C. Gonzalez-Garcia and Y. Nir, hep-ph/0202058.

[11] S. F. King, Nucl. Phys. B576, 85 (2000).

[12] S. M. Bilenky, J. Hosek, and S. T. Petcov, Phys. Lett. 94B, 495 (1980).

[13] B. T. Cleveland et al., Nucl. Phys. B (Proc. Suppl.) 38, 47 (1995); Astrophys. J. 496, 505 (1998).

[14] SAGE Collaboration, J. N. Abdurashitov et al., Phys. Rev. Lett. 83, 4686 (1999); Phys. Rev. C 60, 055801 (1999).

[15] GALLEX Collaboration, W. Hampel et al., Phys. Lett. B 447, 127 (1999).
[16] SNO Collaboration, Q. R. Ahmad et al., Phys. Rev. Lett. 87, 071301 (2001).

[17] SNO Collaboration, Q. R. Ahmad et al., Phys. Rev. Lett. 89, 011301 (2002).

[18] P. Osland and T. T. Wu, report CERN-TH/99-285, hep-ph/0006185; Phys. Scr. T93, 37 (2001).

[19] C. Jarlskog, Phys. Rev. Lett. 55, 1039 (1985).

[20] BABAR Collaboration, P. F. Harrison and H. R. Quinn, SLACR-0504, Papers from Workshop on Physics at an Asymmetric B Factory (BaBar Collaboration Meeting), Rome, Italy, 1996, Princeton, New Jersey, 1997, Orsay, France, 1997, and Pasadena, California, 1997.

[21] J. H. Christenson, J. W. Cronin, V. L. Fitch, and R. Turlay, Phys. Rev. Lett. 13, 138 (1964).

[22] T. T. Wu and C. N. Yang, Phys. Rev. Lett. 13, 380 (1964).

[23] L. Wolfenstein, Phys. Rev. D 17, 2369 (1978); S. P. Mikheev and A. Y. Smirnov, Sov. J. Nucl. Phys. 42, 913 (1985) [Yad. Fiz. 42, 1441 (1985)]; Nuovo Cimento Soc. Ital. Fis., C 9, 17 (1986).

[24] P. Osland and T. T. Wu, Phys. Rev. D 62, 013008 (2000).

[25] Bateman Manuscript Project, Higher Transcendental Functions, edited by A. Erdélyi (McGraw-Hill, New York, 1953), Vol. I.

[26] S. T. Petcov, Phys. Lett. B 200, 373 (1988).

[27] J. N. Bahcall, M. H. Pinsonneault, and S. Basu, Astrophys. J. 555, 990 (2001).

[28] J. N. Bahcall, E. Lisi, D. E. Alburger, L. De Braeckeleer, S. J. Freedman, and J. Napolitano, Phys. Rev. C 54, 411 (1996); J. N. Bahcall, ibid. 56, 3391 (1997); J. N. Bahcall and R. K. Ulrich, Rev. Mod. Phys. 60, 297 (1988); J. N. Bahcall, M. Kamionkowski, and A. Sirlin, Phys. Rev. D 51, 6146 (1995); see also J. N. Bahcall's homepage, http://www.sns.ias.edu/ $\sim$ jnb.

[29] Super-Kamiokande Collaboration, Y. Fukuda et al., Phys. Rev. Lett. 82, 2430 (1999); S. Fukuda et al., ibid. 86, 5656 (2001).

[30] P. Osland and G. Vigdel, Phys. Lett. B 488, 329 (2000).

[31] N. Hata and P. Langacker, Phys. Rev. D 48, 2937 (1993); 50, 632 (1994).

[32] M. C. Gonzalez-Garcia, M. Maltoni, C. Peña-Garay, and J. W. Valle, Phys. Rev. D 63, 033005 (2001).

[33] G. L. Fogli, E. Lisi, A. Marrone, D. Montanino, and A. Palazzo, hep-ph/0104221.

[34] CHOOZ Collaboration, M. Apollonio et al., Phys. Lett. B 420, 397 (1998); 466, 415 (1999).

[35] Y. Koide and H. Fusaoka, Phys. Rev. D 66, 113004 (2002).

[36] C. Giunti and M. Tanimoto, Phys. Rev. D 66, 113006 (2002). 